\title{
Hybrid Approach for Synthesis and Antimicrobial Activity of Heterocyclic Compounds
}

\author{
Nisheeth C. Desai* and Darshita V. Vaja \\ Division of Medicinal Chemistry, Department of Chemistry (DST-FIST Sponsored), \\ Mahatma Gandhi Campus, \\ Maharaja Krishnakumarsinhji Bhavnagar University, Bhavnagar-364002, Gujarat, India \\ dnisheeth@rediffmail.com*
}

Keywords: Anti fungal, antibacterial, microbial strains, spectroscopic techniques.

\begin{abstract}
We have synthesized novel series of $N$-(1-(2-(1-phenyl-3-( $p$-tolyl)-1H-pyrazol-4-yl)-5(pyridin-4-yl)-1,3,4-oxadiazol-3(2H)-yl)ethylidene)arylaniline and their derivatives. The structures of synthesized compounds were well characterized by spectroscopic techniques. Antimicrobial activity of the newly synthesized derivatives was evaluated against gram positive ( $S$. aureus and $S$. pyogenes), gram negative bacteria (E. coli and P. aeruginosa), and strains of fungi (C. albicans, $A$. niger and A. clavatus). Among the screened derivatives 5c, 5f, 5i, $\mathbf{5 l}$ and $\mathbf{5 t}$ demonstrated superior antimicrobial activity against microbial strains.
\end{abstract}

\section{Introduction}

Nitrogen-rich heterocycles, particularly pyrazole 1,3,4-oxadiazole and pyridine, represent an exclusive class of diversified frameworks exhibiting a broad spectrum of biological functions. Some microbial species are responsible for dangerous diseases such as food poisoning, typhoid and due to this; it is the global concern for public health. All these species are fully resisted against old fashionable antimicrobial drugs. For this reason new discovery of antimicrobials are important for the medicinal chemists. Heterocycles comprising nitrogen especially five member rings have captured huge interest due to their availability in many natural products and as vital part of synthetic bioactive molecules [1]. In this framework, pyridine, pyrazole and 1,3,4-oxadiazole templates featuring one, two nitrogen's and an oxygen atom [2] is recognized as a member of fortunate structural class particularly from medicinal point of view [3]. Commercially available drugs (Fig. 1) celecoxibe, zibotentan and sulphapyridine sequentially contain pyrazole [4], 1,3,4-oxadiazole [5] and pyridine heterocycles. Among the important heterocyclic scaffolds of biological and pharmacological interest, the pyrazole ring is able with diverse medicinal activities [6-12].

1,3,4-oxadiazoles scaffold is a group of heterocyclic compounds as they have attracted significant concern in medicinal chemistry. 1,3,4-oxadiazoles displayed a significant biological activity such as, antimicrobial [13], anti-HIV [14], antitumor [15], anticonvulsant [16], anti tubercular [17], antihypertensive. The pyridine core is leading in several natural products and significant important in the medicinal chemistry. A large number of biological activities are related with pyridines derivatives such as, anticancer [18], anti-ulcer [19], antitubercular and antimicrobial [20] activities etc.

Molecular hybridization is a significant tool for discovery of novel chemical entities. In the past some decades much attention has been given to the plan and synthesis of new types of pharmacologically diverse structural hybrid molecules [21]. Encouraged by the above-mentioned explanation, we have focused onto construct some novel antimicrobial derivatives containing pyrazole, 1,3,4-oxadiazole and pyridine scaffolds screened for antimicrobial activity. Previously our research group has worked on the synthesis of pyrazole and their derivatives as potential antimicrobials [22-24]. In furtherance to this, $N$-(1-(2-(1-phenyl-3-( $p$-tolyl)-1H-pyrazol-4-yl)-5(pyridin-4-yl)-1,3,4-oxadiazol-3(2H)-yl)ethylidine)arylanilines (5a-t) have been synthesized and screened for their antimicrobial activity against standard strains of bacteria and fungi [25]. All final 
compounds were categorised by different spectroscopic methods such as, infrared spectroscopy, ${ }^{1} \mathrm{H}$ and ${ }^{13} \mathrm{C}$ nuclear magnetic resonance and mass.

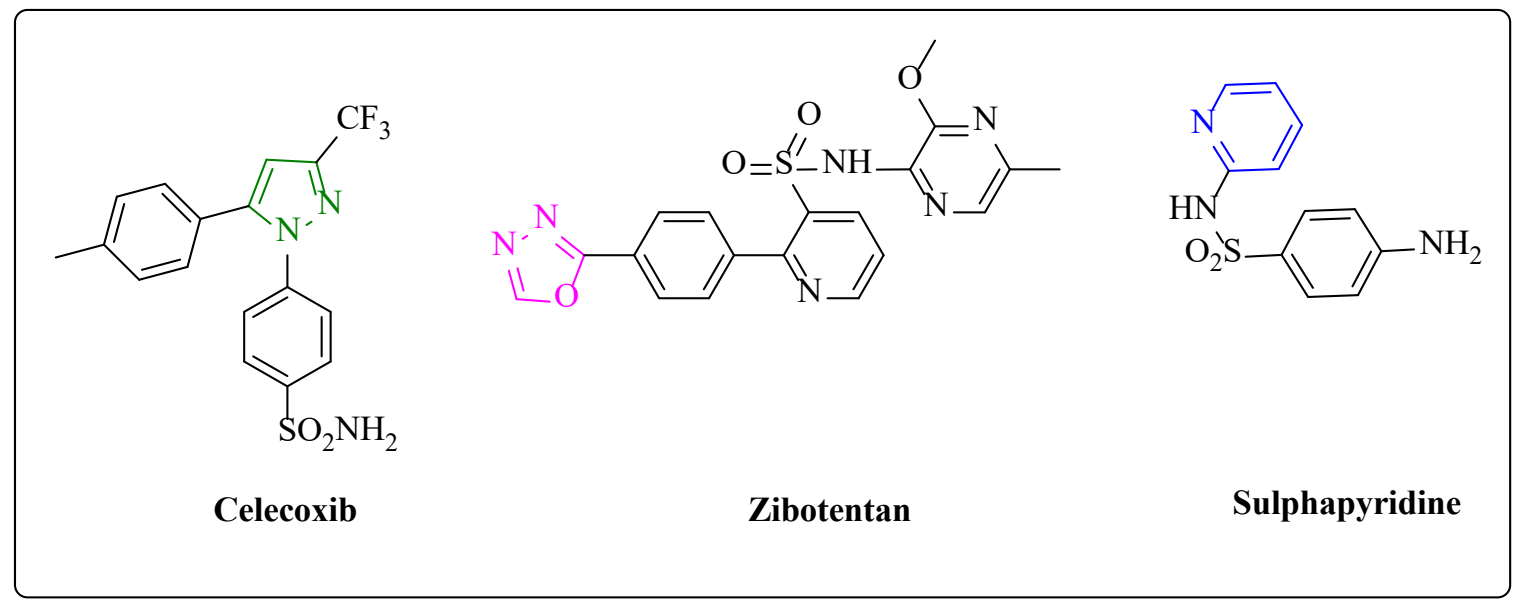

Figure 1. Chemical structures of clinically used antimicrobial agents.

\section{Results and Discussion}

\section{Chemistry}

All reactions except those in aqueous media were carried out by standard techniques for the keeping out of wetness. Melting points were determined on an electro thermal melting point apparatus and were reported uncorrected. TLC on silica gel plates (Merck, 60, $\mathrm{F}_{254}$ ) was used for purity checking and reaction monitoring. Column chromatography on silica gel (Merck, 70-230 mesh and 230-400 mesh ASTH for flash chromatography) was applied when necessary to isolate and purify the reaction products. Elemental analysis $(\% \mathrm{C}, \mathrm{H}, \mathrm{N})$ was carried out by a Perkin-Elmer $2400 \mathrm{CHN}$ analyzer. IR spectra of all compounds were recorded on a Perkin-Elmer FT-IR spectrophotometers in $\mathrm{KBr} .{ }^{1} \mathrm{H}$ NMR and ${ }^{13} \mathrm{C}$ NMR spectra were recorded on Varian Gemini $500 \mathrm{MHz}$ in DMSO-d $\mathrm{d}_{6}$ as a solvent and tetramethylsilane (TMS) as an internal standard. Mass spectra were scanned on a Shimadzu LC-MS 2010 spectrometer. Anhydrous reactions were carried out in oven-dried glassware in nitrogen atmosphere.

Synthetic tactics adopted to get the final compounds are depicted in Scheme 1. Here, compound 5 is a part of synthesis of new chemical moieties in the form of antimicrobials. In scheme 1, the key Schiff base $N^{\prime}$-((1-phenyl-3-(p-tolyl)-1H-pyrazol-4yl)methylene)isonicotinohydrazide (3) was used as a starting material for the synthesis of final compounds (5a-t). Compound (3) was synthesized through simple condensation by 1-phenyl-3-( $p$ tolyl)-1H-pyrazole-4-carbaldehyde (1) and double amount of Isoniazide in 1,4-dioxane used as solvent. Compound (1) is Vilsmeier-Haack synthesis product; it provides intermediate for the synthesis of some novel substituted heterocyclic moieties. Compound (3) was cyclised by acetic anhydride and yielded 1-(2-(1-phenyl-3-( $p$-tolyl)-1H-pyrazol-4-yl)-5-(pyridin-4-yl)-1,3,4oxadiazol-3(2H)-yl)ethanone (4). Further in final step compound (4) and different substituted aromatic anilines derivatives were condensed and convert in final products (5a-t).

Novel compounds (5a-t) were categorized by different spectroscopic methods such as, IR, ${ }^{1} \mathrm{H}$ $\mathrm{NMR},{ }^{13} \mathrm{C} \mathrm{NMR}$, and mass. In compound 51, group $>\mathrm{C}=\mathrm{N}-$ and $>\mathrm{C}=\mathrm{C}<$ stretching vibrations appeared at 1597 and $1537 \mathrm{~cm}^{-1}$. Nitro group of aniline showed stretching vibrations at $1504 \mathrm{~cm}^{-1}$. $\mathrm{C}-\mathrm{CH}_{3}$ group which was attached to 1,3,4-oxadiazole ring showed stretching vibrations at $1355 \mathrm{~cm}^{-1}$. The absorption band at 1292 and $1224 \mathrm{~cm}^{-1}$ is due to stretching vibration of $>\mathrm{C}=\mathrm{N}-$ and C-O-C oxadiazole ring. In ${ }^{1} \mathrm{H}$ NMR characterization $\mathrm{C}_{2}-\mathrm{H}$ and $\mathrm{C}_{6}-\mathrm{H}$ protons of pyridine ring showed doublet at $8.82 \mathrm{ppm}$. In pyridine ring two protons $\mathrm{C}_{3}-\mathrm{H}$ and $\mathrm{C}_{5}-\mathrm{H}$ appeared at $8.12 \mathrm{ppm}$ as doublet. Singlet signal of pyrazole ring was given by $\mathrm{C}_{5}-\mathrm{H}$ at $7.96 \mathrm{ppm}$. All other aromatic 13 protons showed multiple in range of $6.95-8.15 \mathrm{ppm}$. At $5.85 \mathrm{ppm}$ singlet signals appeared by one 
proton of oxadiazole ring. Remaining six protons showed two singlet signals at 2.39 and $2.28 \mathrm{ppm}$ out of six three protons are of $-\mathrm{CH}_{3}$ ring and another three from $-\mathrm{CH}_{3}-\mathrm{C}=\mathrm{N}$. Looking to the ${ }^{13} \mathrm{C}$ NMR spectra, the chemical shifts of the final compound $\mathbf{5 l}$ has carbons that varied from $\delta=158.3$ $21.5 \mathrm{ppm}$. Signal of $\mathrm{C}_{2}$ and $\mathrm{C}_{5}$ in 1,3,4-oxadiazole ring appeared at 158.0 at and $78.3 \mathrm{ppm}$. In pyridine ring $\mathrm{C}_{2}, \mathrm{C}_{3}$ and $\mathrm{C}_{5}$ showed peak at $149.2,138.2$ and $124.7 \mathrm{ppm}$. In pyrazole ring system $\mathrm{C}_{3}, \mathrm{C}_{4}$ and $\mathrm{C}_{5}$ signals appeared at $149.5,123.8$ and $117.8 \mathrm{ppm}$. One carbon of $-\underline{\mathrm{C}} \mathrm{H}=\mathrm{N}$ - displayed peak at $156.3 \mathrm{ppm}$. Furthermore, the mass spectrum of (5a-t) appeared a molecular ion peak corresponding to molecular formula (5a-t) onward of other fragment peaks, which supported the proposed structure of final compounds. Detail discussion of experimental data is given in supporting section.

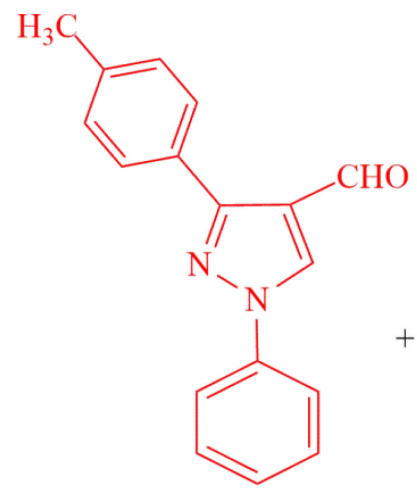

(1)<smiles>CC(=N[14C])N1N=C(c2ccncc2)OC1c1cn(-c2ccccc2)nc1-c1ccc(C)cc1</smiles>

(5)

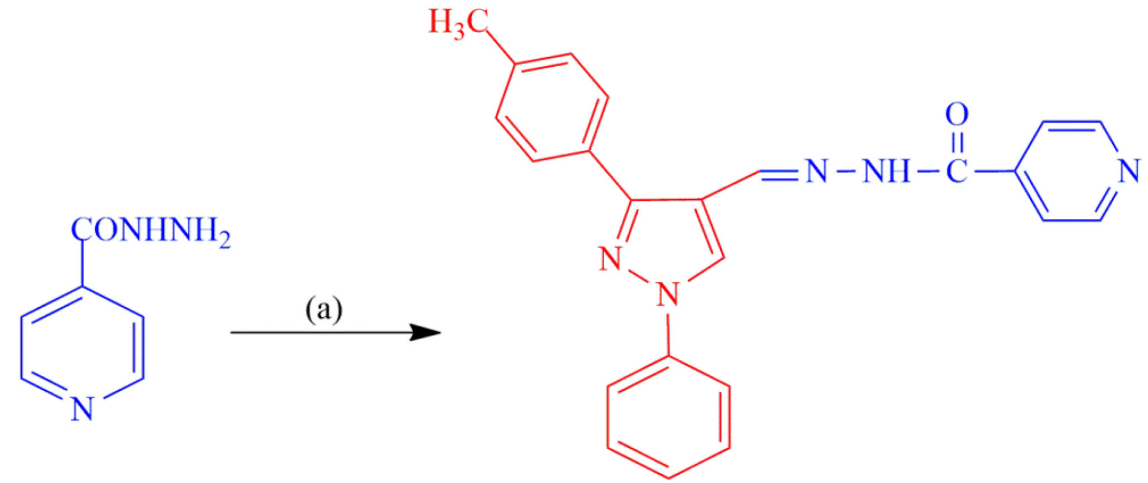

(3)

(b) $\left(\mathrm{CH}_{3} \mathrm{CO}\right)_{2} \mathrm{O}$ $\mathrm{a}=1,4-$ Dioxan, $4 \mathrm{~h}$

$\mathrm{b}=$ Reflux, 6h,

$\mathrm{c}=$ Ethanol, HAc, $12 \mathrm{~h}$

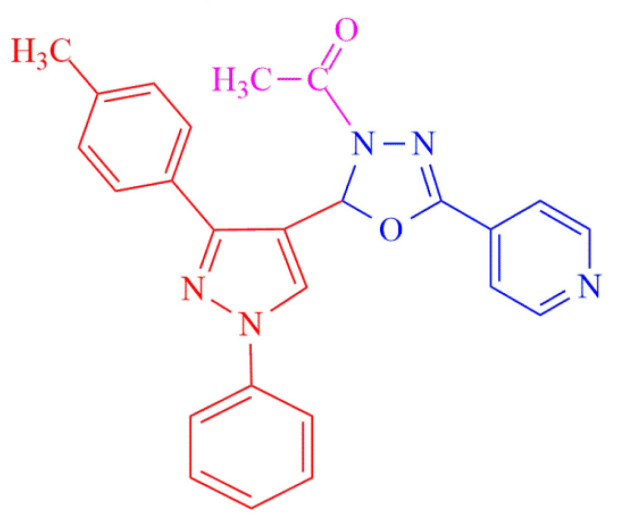

(4)

$$
\begin{aligned}
& \mathrm{Ar}=\mathrm{C}_{6} \mathrm{H}_{5}-\mathrm{H}, \quad \mathrm{C}_{6} \mathrm{H}_{4}-4-\mathrm{CH}_{3}, \quad \mathrm{C}_{6} \mathrm{H}_{4}-3-\mathrm{NO}_{2}, \quad \mathrm{C}_{6} \mathrm{H}_{4}-2-\mathrm{Br} \\
& \mathrm{C}_{6} \mathrm{H}_{4}-2-\mathrm{F}, \quad \mathrm{C}_{6} \mathrm{H}_{4}-2-\mathrm{OCH}_{3}, \quad \mathrm{C}_{6} \mathrm{H}_{4}-4-\mathrm{NO}_{2}, \quad \mathrm{C}_{6} \mathrm{H}_{4}-3-\mathrm{Br} \\
& \mathrm{C}_{6} \mathrm{H}_{4}-4-\mathrm{F}, \quad \mathrm{C}_{6} \mathrm{H}_{4}-3-\mathrm{OCH}_{3}, \quad \mathrm{C}_{6} \mathrm{H}_{4}-2-\mathrm{Cl}, \quad \mathrm{C}_{6} \mathrm{H}_{4}-4-\mathrm{Br} \\
& \mathrm{C}_{6} \mathrm{H}_{4}-2-\mathrm{CH}_{3}, \mathrm{C}_{6} \mathrm{H}_{4}-4-\mathrm{OCH}_{3}, \quad \mathrm{C}_{6} \mathrm{H}_{4}-3-\mathrm{Cl}, \quad \mathrm{C}_{6} \mathrm{H}_{3}-4-\mathrm{Br}-2-\mathrm{CH}_{3} \\
& \mathrm{C}_{6} \mathrm{H}_{4}-3-\mathrm{CH}_{3}, \quad \mathrm{C}_{6} \mathrm{H}_{4}-2-\mathrm{NO}_{2}, \quad \mathrm{C}_{6} \mathrm{H}_{4}-4-\mathrm{Cl}, \quad \mathrm{C}_{6} \mathrm{H}_{3}-3-\mathrm{Cl}-4-\mathrm{F}
\end{aligned}
$$

Scheme 1. Synthetic pathway of targeted compounds (5a-t).

Preparation of $N^{\prime}$-((1-phenyl-3-(p-tolyl)-1H-pyrazol-4-yl)methylene)isonicotinohydrazide (3) and preparation of 1-(2-(1-phenyl-3-(p-tolyl)-1H-pyrazol-4-yl)-5-(pyridin-4-yl)-1,3,4oxadiazol-3(2H)-yl)ethanone. (4)

Synthesis of compound (3) and (4) were achieved by reported method [26]. 


\section{Preparation of $\mathrm{N}$-(1-(2-(1-phenyl-3-(p-tolyl)-1 H-pyrazol-4-yl)-5-(pyridin-4-yl)-1,3,4-oxadiazol- $3(2 H)$-yl)ethylidene)arylaniline. (5)}

A mixture of compound 1-(2-(1-phenyl-3-(p-tolyl)-1H-pyrazol-4-yl)-5-(pyridin-4-yl)-1,3,4oxadiazol-3(2H)-yl)ethanone (4) $(0.01 \mathrm{~mol})$ and substituted aniline derivatives $(0.02 \mathrm{~mol})$ were taken in $30 \mathrm{~mL}$ ethanol $(95 \%)$ and refluxed at $120^{\circ} \mathrm{C}$ for the period of $12 \mathrm{~h}$. The mixture was concentrated under vacuum and poured into ice cold water. The solid products was separated and recrystalised in ethanol (95\%). The completion of the reaction and the purity of compounds (3), (4) and (5) were checked on TLC [Aluminium sheet-silica gel 60, $\mathrm{F}_{245}$ (E. Merck)] using hexane-ethyl acetate $(8: 2 \mathrm{v} / \mathrm{v})$ as an irrigator and were developed in iodine chamber.

Table 1. Physical constant of derivatives (5a-t).

\begin{tabular}{|c|c|c|c|c|}
\hline $\begin{array}{l}\text { Sr. } \\
\text { no }\end{array}$ & Substitution & $\begin{array}{l}\text { M.P. } \\
\left({ }^{\circ} \mathrm{C}\right)\end{array}$ & $\begin{array}{l}\text { Yield } \\
\text { (\%) }\end{array}$ & Colour \\
\hline $\mathbf{5 a}$ & $\mathrm{C}_{6} \mathrm{H}_{4}-\mathrm{H}$ & $177-180^{\circ} \mathrm{C}$ & 63 & Creemish yellow \\
\hline $5 \mathbf{b}$ & $\mathrm{C}_{6} \mathrm{H}_{4}-2-\mathrm{F}$ & $145-148^{\circ} \mathrm{C}$ & 70 & Off white \\
\hline $5 c$ & $\mathrm{C}_{6} \mathrm{H}_{4}-4-\mathrm{F}$ & $135-138^{\circ} \mathrm{C}$ & 79 & Yellowish white \\
\hline $5 d$ & $\mathrm{C}_{6} \mathrm{H}_{4}-2-\mathrm{CH}_{3}$ & $120-122^{\circ} \mathrm{C}$ & 64 & Light yellow \\
\hline $5 e$ & $\mathrm{C}_{6} \mathrm{H}_{4}-3-\mathrm{CH}_{3}$ & $156-158^{\circ} \mathrm{C}$ & 66 & Yellow \\
\hline $5 f$ & $\mathrm{C}_{6} \mathrm{H}_{4}-4-\mathrm{CH}_{3}$ & $202-204^{\circ} \mathrm{C}$ & 71 & Dark yellow \\
\hline $5 g$ & $\mathrm{C}_{6} \mathrm{H}_{4}-2-\mathrm{OCH}_{3}$ & $158-159^{\circ} \mathrm{C}$ & 64 & Off white \\
\hline $5 \mathrm{hh}$ & $\mathrm{C}_{6} \mathrm{H}_{4}-3-\mathrm{OCH}_{3}$ & $185-187^{\circ} \mathrm{C}$ & 69 & Creemish \\
\hline $5 \mathbf{i}$ & $\mathrm{C}_{6} \mathrm{H}_{4}-4-\mathrm{OCH}_{3}$ & $110-112^{\circ} \mathrm{C}$ & 67 & Buff \\
\hline $5 \mathbf{j}$ & $\mathrm{C}_{6} \mathrm{H}_{4}-2-\mathrm{NO}_{2}$ & $112-114^{\circ} \mathrm{C}$ & 56 & Yellow \\
\hline $5 k$ & $\mathrm{C}_{6} \mathrm{H}_{4}-3-\mathrm{NO}_{2}$ & $165-167^{\circ} \mathrm{C}$ & 59 & Dark yellow \\
\hline 51 & $\mathrm{C}_{6} \mathrm{H}_{4}-4-\mathrm{NO}_{2}$ & $126-129^{\circ} \mathrm{C}$ & 60 & Orange \\
\hline $5 \mathrm{~m}$ & $\mathrm{C}_{6} \mathrm{H}_{4}-2-\mathrm{Cl}$ & $140-142^{\circ} \mathrm{C}$ & 62 & White \\
\hline $5 n$ & $\mathrm{C}_{6} \mathrm{H}_{4}-3-\mathrm{Cl}$ & $196-198^{\circ} \mathrm{C}$ & 66 & Off white \\
\hline 50 & $\mathrm{C}_{6} \mathrm{H}_{4}-4-\mathrm{Cl}$ & $204-206^{\circ} \mathrm{C}$ & 68 & Brown \\
\hline $5 p$ & $\mathrm{C}_{6} \mathrm{H}_{4}-2-\mathrm{Br}$ & $196-199^{\circ} \mathrm{C}$ & 70 & Yellow \\
\hline $5 q$ & $\mathrm{C}_{6} \mathrm{H}_{4}-3-\mathrm{Br}$ & $177-179^{\circ} \mathrm{C}$ & 72 & Brown \\
\hline $5 r$ & $\mathrm{C}_{6} \mathrm{H}_{4}-4-\mathrm{Br}$ & $188-190^{\circ} \mathrm{C}$ & 75 & Reddish brown \\
\hline $5 s$ & $\mathrm{C}_{6} \mathrm{H}_{3}-4-\mathrm{Br}-2-\mathrm{CH}_{3}$ & $162-164^{\circ} \mathrm{C}$ & 65 & Off white \\
\hline $5 t$ & $\mathrm{C}_{6} \mathrm{H}_{3}-3-\mathrm{Cl}-4-\mathrm{F}$ & $171-173^{\circ} \mathrm{C}$ & 63 & Pale yellow \\
\hline
\end{tabular}

Characterization of $\quad N$-(1-(2-(1-phenyl-3-(p-tolyl)-1 $H$-pyrazol-4-yl)-5-(pyridin-4-yl)-1,3,4oxadiazol-3(2H)-yl)ethylidene)aniline: 5 a

IR $\left(\mathrm{KBr}, \mathrm{cm}^{-1}\right): 1588,1543\left(>\mathrm{C}=\mathrm{N}-,>\mathrm{C}=\mathrm{C}<\right.$ stretching), $1372\left(-\mathrm{C}-\mathrm{H}\right.$ stretching, $\left.-\mathrm{C}-\mathrm{CH}_{3}\right)$, $1295\left(>\mathrm{C}=\mathrm{N}\right.$ - stretching, Ar- $\left.\mathrm{NH}_{2}\right), 1230$ (C-O-C stretching, oxadiazole ring); ${ }^{1} \mathrm{H}$ NMR (DMSO- $d_{6}$, $400 \mathrm{MHz}): \delta=8.96\left(\mathrm{~d}, 2 \mathrm{H}, J=8.1 \mathrm{~Hz}, \mathrm{C}_{2}-\mathrm{H}\right.$ and $\mathrm{C}_{6}-\mathrm{H}$ pyridine ring $), 8.0\left(\mathrm{~d}, 2 \mathrm{H}, J=7.8 \mathrm{~Hz}, \mathrm{C}_{3}-\mathrm{H}\right.$ and $\mathrm{C}_{5}-\mathrm{H}$ pyridine ring), $7.96\left(\mathrm{~s}, 1 \mathrm{H}, \mathrm{C}_{5}-\mathrm{H}\right.$ pyrazole ring), 6.94-7.75 (m, 14H, Ar-H), $5.70(\mathrm{~s}, 1 \mathrm{H}$, $\mathrm{C}_{5}$ - $\mathrm{H}$ oxadiazole ring), $2.40\left(\mathrm{~s}, 3 \mathrm{H},-\mathrm{CH}_{3}\right), 2.20\left(\mathrm{~s}, 3 \mathrm{H},-\mathrm{CH}_{3}-\mathrm{C}=\mathrm{N}\right) ;{ }^{13} \mathrm{C}$ NMR $(100 \mathrm{MHz}, \mathrm{DMSO}-$ $\left.d_{6}\right): \delta=157.5\left(1 \mathrm{C}, \mathrm{C}_{5}\right.$ oxadiazole ring), $156.1(1 \mathrm{C},-\mathrm{CH}=\mathrm{N}-), 152.8\left(1 \mathrm{C}, \mathrm{C}_{1} \mathrm{C}_{5} \mathrm{H}_{5}-\mathrm{N}=\right), 149.0(1 \mathrm{C}$, $\mathrm{C}_{3}$ pyrazole ring), $149.9\left(2 \mathrm{C}, \mathrm{C}_{2}\right.$ and $\mathrm{C}_{6}$ pyridine ring), $139.4\left(1 \mathrm{C}, \mathrm{C}_{1}\right.$ aromatic ring), $138.5\left(1 \mathrm{C}, \mathrm{C}_{4}\right.$ pyridine ring), $131.4\left(1 \mathrm{C}, \mathrm{C}_{1} \mathrm{C}_{6} \mathrm{H}_{4}-\mathrm{CH}_{3}\right), 130.8\left(3 \mathrm{C}, \mathrm{C}_{3}\right.$ and $\left.\mathrm{C}_{5} \mathrm{C}_{5} \mathrm{H}_{5}-\mathrm{N}=, \mathrm{C}_{1} \mathrm{C}_{6} \mathrm{H}_{4}-\mathrm{CH}_{3}\right), 129.6(2 \mathrm{C}$, $\mathrm{C}_{3}$ and $\left.\mathrm{C}_{5} \mathrm{C}_{6} \mathrm{H}_{4}-\mathrm{CH}_{3}\right), 129.1\left(2 \mathrm{C}, \mathrm{C}_{3}\right.$ and $\mathrm{C}_{5}$ aromatic ring), $127.0\left(1 \mathrm{C}, \mathrm{C}_{4}-\mathrm{C}_{5} \mathrm{H}_{5}-\mathrm{N}=\right), 126.9\left(1 \mathrm{C}, \mathrm{C}_{4}\right.$ aromatic ring), $125.2\left(2 \mathrm{C}, \mathrm{C}_{2}\right.$ and $\left.\mathrm{C}_{6} \mathrm{C}_{6} \mathrm{H}_{4}-\mathrm{CH}_{3}\right), 124.4\left(2 \mathrm{C}, \mathrm{C}_{3}\right.$ and $\mathrm{C}_{5}$ pyridine ring), 123.5 (1C, $\mathrm{C}_{5}$ pyrazole ring), $122.9\left(2 \mathrm{C}, \mathrm{C}_{2}\right.$ and $\left.\mathrm{C}_{6} \mathrm{C}_{5} \mathrm{H}_{5}-\mathrm{N}=\right), 119.3$ (2C, $\mathrm{C}_{2}$ and $\mathrm{C}_{6}$ aromatic ring), $117.7\left(1 \mathrm{C}, \mathrm{C}_{4}\right.$ pyrazole ring), 78.2 (1C, $\mathrm{C}_{2}$ oxadiazole ring), 29.6 (1C, $\mathrm{C}_{5}$ aromatic ring), $21.5\left(1 \mathrm{C}, \mathrm{C}_{6} \mathrm{H}_{4}-\mathrm{CH}_{3}\right)$, $21.1\left(1 \mathrm{C},=\mathrm{CH}-\mathrm{CH}_{3}\right)$; LCMS (ESI): $m / z 499.2(36.1 \%)\left[\mathrm{M}^{+}\right]$; Anal. calcd. for $\mathrm{C}_{31} \mathrm{H}_{26} \mathrm{~N}_{6} \mathrm{O}: \mathrm{C}, 74.68$; H, 5.26; N, 16.86; Found: C, 74.72; H, 5.33; N, 16.93\%. 


\section{Characterization of 2-fluoro- $N$-(1-(2-(1-phenyl-3-(p-tolyl)-1H-pyrazol-4-yl)-5-(pyridin-4-yl)- 1,3,4-oxadiazol-3(2H)-yl)ethylidene)aniline: $5 \mathrm{~b}$}

IR $\left(\mathrm{KBr}, \mathrm{cm}^{-1}\right): 1582,1546\left(>\mathrm{C}=\mathrm{N}-,>\mathrm{C}=\mathrm{C}<\right.$ stretching), $1374\left(-\mathrm{C}-\mathrm{H}\right.$ stretching, $\left.-\mathrm{C}-\mathrm{CH}_{3}\right)$, $1293\left(>\mathrm{C}=\mathrm{N}\right.$ - stretching, Ar-NH $\left.{ }_{2}\right), 1233$ (C-O-C stretching, oxadiazole ring), 1075 (C-F stretching); ${ }^{1} \mathrm{H}$ NMR (DMSO- $\left.\mathrm{d}_{6}, 400 \mathrm{MHz}\right): \delta=8.92\left(\mathrm{~d}, 2 \mathrm{H}, J=8.3 \mathrm{~Hz} \mathrm{C}_{2}-\underline{\mathrm{H}}\right.$ and $\mathrm{C}_{6}-\underline{\mathrm{H}}$ pyridine ring), $8.02(\mathrm{~d}$, $2 \mathrm{H}, J=7.2 \mathrm{~Hz}, \mathrm{C}_{3}-\underline{\mathrm{H}}$ and $\mathrm{C}_{5}-\underline{\mathrm{H}}$ pyridine ring), $7.93\left(\mathrm{~s}, 1 \mathrm{H}, \mathrm{C}_{5}-\underline{\mathrm{H}}\right.$ pyrazole ring), 7.28-7.79 (m, 13H, $\mathrm{Ar}-\underline{\mathrm{H}}), 5.72\left(\mathrm{~s}, 1 \mathrm{H}, \mathrm{C}_{5}-\underline{\mathrm{H}}\right.$ oxadiazole ring), $2.41\left(\mathrm{~s}, 3 \mathrm{H},-\mathrm{CH}_{3}\right), 2.22\left(\mathrm{~s}, 3 \mathrm{H},-\mathrm{CH}_{3}-\mathrm{C}=\mathrm{N}\right) ;{ }^{13} \mathrm{C} \mathrm{NMR}$ $\left(100 \mathrm{MHz}, \mathrm{DMSO}-\mathrm{d}_{6}\right): \bar{\delta}=157.5\left(1 \mathrm{C}, \mathrm{C}_{5}\right.$ oxadiazole ring), $156.4(1 \mathrm{C},-\mathrm{CH}=\mathrm{N}-), 153.0(1 \mathrm{C}, \mathrm{JC}-\mathrm{F}$ $\left.2367 \mathrm{c} / \mathrm{sec}, \mathrm{C}_{2} \mathrm{C}_{5} \mathrm{H}_{4}-\mathrm{N}=\right), 149.5\left(2 \mathrm{C}, \mathrm{C}_{2}\right.$ and $\mathrm{C}_{6}$ pyridine ring), 149.1 (1C, $\mathrm{C}_{3}$ pyrazole ring), 139.3 (1C, $\mathrm{C}_{1}$ aromatic ring), $138.6\left(1 \mathrm{C}, \mathrm{C}_{4}\right.$ pyridine ring), $136.5\left(1 \mathrm{C}, \mathrm{C}_{1} \mathrm{C}_{5} \mathrm{H}_{5}-\mathrm{N}=\right), 131.8\left(1 \mathrm{C}, \mathrm{C}_{4} \mathrm{C}_{6} \mathrm{H}_{4^{-}}\right.$ $\left.\mathrm{CH}_{3}\right), 130.5\left(1 \mathrm{C}, \mathrm{C}_{1} \mathrm{C}_{6} \mathrm{H}_{4}-\mathrm{CH}_{3}\right), 129.9\left(2 \mathrm{C}, \mathrm{C}_{3}\right.$ and $\left.\mathrm{C}_{5} \mathrm{C}_{6} \mathrm{H}_{4}-\mathrm{CH}_{3}\right), 129.1$ (2C, $\mathrm{C}_{3}$ and $\mathrm{C}_{5}$ aromatic ring), $128.4\left(1 \mathrm{C}, \mathrm{C}_{4} \mathrm{C}_{5} \mathrm{H}_{4}-\mathrm{N}=\right), 126.7$ (1C, $\mathrm{C}_{4}$ aromatic ring), $125.3\left(2 \mathrm{C}, \mathrm{C}_{2}\right.$ and $\left.\mathrm{C}_{6} \mathrm{C}_{6} \mathrm{H}_{4}-\mathrm{CH}_{3}\right)$, $125.7\left(1 \mathrm{C}, \mathrm{C}_{5} \mathrm{C}_{5} \mathrm{H}_{5}-\mathrm{N}=\right), 124.8$ (2C, $\mathrm{C}_{3}$ and $\mathrm{C}_{5}$ pyridine ring), $123.7\left(1 \mathrm{C}, \mathrm{C}_{6} \mathrm{C}_{5} \mathrm{H}_{5}-\mathrm{N}=\right), 123.4(1 \mathrm{C}$, $\mathrm{C}_{5}$ pyrazole ring), $119.7\left(2 \mathrm{C}, \mathrm{C}_{2}\right.$ and $\mathrm{C}_{6}$ aromatic ring), $118.0\left(1 \mathrm{C}, \mathrm{C}_{3} \mathrm{C}_{5} \mathrm{H}_{5}-\mathrm{N}=\right), 117.8\left(1 \mathrm{C}, \mathrm{C}_{4}\right.$ pyrazole ring), $78.6\left(1 \mathrm{C}, \mathrm{C}_{2}\right.$ oxadiazole ring), $21.8\left(1 \mathrm{C}, \mathrm{C}_{6} \mathrm{H}_{4}-\mathrm{CH}_{3}\right), 21.5\left(1 \mathrm{C},=\mathrm{CH}-\mathrm{CH}_{3}\right)$; LCMS (ESI): $\mathrm{m} / \mathrm{z} 517.2(36.1 \%)\left[\mathrm{M}^{+}\right]$; Anal. calcd. for $\mathrm{C}_{31} \mathrm{H}_{25} \mathrm{FN}_{6} \mathrm{O}: \mathrm{C}, 72.08 ; \mathrm{H}, 4.88 ; \mathrm{N}, 16.27$; Found: C, $72.15 ; \mathrm{H}, 4.92 ; \mathrm{N}, 16.30 \%$.

\section{Characterization of 4-fluoro- $N$-(1-(2-(1-phenyl-3-(p-tolyl)-1 $H$-pyrazol-4-yl)-5-(pyridin-4-yl)- 1,3,4-oxadiazol-3( $2 \mathrm{H})$-yl)ethylidene)aniline: $5 \mathrm{c}$}

IR $\left(\mathrm{KBr}, \mathrm{cm}^{-1}\right): 1587,1540\left(>\mathrm{C}=\mathrm{N}-,>\mathrm{C}=\mathrm{C}<\right.$ stretching), $1379\left(-\mathrm{C}-\mathrm{H}\right.$ stretching, $\left.-\mathrm{C}-\mathrm{CH}_{3}\right)$, $1296\left(>\mathrm{C}=\mathrm{N}\right.$ - stretching, Ar- $\left.\mathrm{NH}_{2}\right), 1238$ (C-O-C stretching, oxadiazole ring), 1050 (C-F stretching); ${ }^{1} \mathrm{H}$ NMR (DMSO- $\left.\mathrm{d}_{6}, 400 \mathrm{MHz}\right): \delta=8.93\left(\mathrm{~d}, 2 \mathrm{H}, J=8.3 \mathrm{~Hz} \mathrm{C}-\underline{\mathrm{H}}\right.$ and $\mathrm{C}_{6}-\underline{\mathrm{H}}$ pyridine ring), 8.03 (d, $2 \mathrm{H}, J=7.3 \mathrm{~Hz} \mathrm{C}_{3}-\underline{\mathrm{H}}$ and $\mathrm{C}_{5}-\underline{\mathrm{H}}$ pyridine ring), 7.99 (s, $1 \mathrm{H}, \mathrm{C}_{5}-\underline{\mathrm{H}}$ pyrazole ring), 7.30-7.85 (m, $13 \mathrm{H}$, Ar- $\underline{\mathrm{H}}), 5.78\left(\mathrm{~s}, 1 \mathrm{H}, \mathrm{C}_{5}-\underline{\mathrm{H}}\right.$ oxadiazole ring), $2.44\left(\mathrm{~s}, 3 \mathrm{H},-\mathrm{CH}_{3}\right), 2.25\left(\mathrm{~s}, 3 \mathrm{H},-\mathrm{CH}_{3}-\mathrm{C}=\mathrm{N}\right) ;{ }^{13} \mathrm{C}$ NMR $\left(100 \mathrm{MHz}, \mathrm{DMSO}-\mathrm{d}_{6}\right): \delta=161.4\left(1 \mathrm{C}, \mathrm{JC}-\mathrm{F} 2333 \mathrm{c} / \mathrm{sec}, \mathrm{C}_{4} \mathrm{C}_{5} \mathrm{H}_{4}-\mathrm{N}=\right), 157.4\left(1 \mathrm{C}, \mathrm{C}_{5}\right.$ oxadiazole ring), $156.5(1 \mathrm{C},-\mathrm{CH}=\mathrm{N}-), 149.6\left(2 \mathrm{C}, \mathrm{C}_{2}\right.$ and $\mathrm{C}_{6}$ pyridine ring), 149.2 ( $1 \mathrm{C}, \mathrm{C}_{3}$ pyrazole ring), 147.5 (1C, $\left.\mathrm{C}_{1} \mathrm{C}_{5} \mathrm{H}_{4}-\mathrm{N}=\right)$ ) 139.5 (1C, $\mathrm{C}_{1}$ aromatic ring), 138.0 (1C, $\mathrm{C}_{4}$ pyridine ring), 131.3, 130.4 (1C, $\mathrm{C}_{1}$ $\left.\mathrm{C}_{6} \mathrm{H}_{4}-\mathrm{CH}_{3}\right), 129.8$ (2C, $\mathrm{C}_{3}$ and $\mathrm{C}_{5} \mathrm{C}_{6} \mathrm{H}_{4}-\mathrm{CH}_{3}$ ), 129.7 (2C, $\mathrm{C}_{3}$ and $\mathrm{C}_{5}$ aromatic ring), 126.4 (1C, $\mathrm{C}_{4}$ aromatic ring), $125.4\left(2 \mathrm{C}, \mathrm{C}_{2}\right.$ and $\left.\mathrm{C}_{6} \mathrm{C}_{6} \mathrm{H}_{4}-\mathrm{CH}_{3}\right), 124.4$ (2C, $\mathrm{C}_{3}$ and $\mathrm{C}_{5}$ pyridine ring), 123.9 (2C, $\mathrm{C}_{6}$ and $\left.\mathrm{C}_{2} \mathrm{C}_{5} \mathrm{H}_{4}-\mathrm{N}=\right), 123.7$ (1C, $\mathrm{C}_{5}$ pyrazole ring), 119.7 (2C, $\mathrm{C}_{2}$ and $\mathrm{C}_{6}$ aromatic ring), 117.5 (1C, $\mathrm{C}_{4}$ pyrazole ring), $116.2\left(2 \mathrm{C}, \mathrm{C}_{5}\right.$ and $\left.\mathrm{C}_{3} \mathrm{C}_{5} \mathrm{H}_{4}-\mathrm{N}=\right)$, $78.1\left(1 \mathrm{C}, \mathrm{C}_{2}\right.$ oxadiazole ring), $21.4\left(1 \mathrm{C}, \mathrm{C}_{6} \mathrm{H}_{4}-\right.$ $\left.\mathrm{CH}_{3}\right), 21.6\left(1 \mathrm{C},=\mathrm{CH}-\mathrm{CH}_{3}\right)$; LCMS (ESI): $\mathrm{m} / \mathrm{z} 517.2(36.1 \%)\left[\mathrm{M}^{+}\right]$; Anal. calcd. for $\mathrm{C}_{31} \mathrm{H}_{25} \mathrm{FN}_{6} \mathrm{O}$ : C, 72.08; H, 4.88; N, 16.27; Found: C,72.12; H, 4.94; N, 16.33\%.

\section{Characterization of 2-methyl- $N$-(1-(2-(1-phenyl-3-(p-tolyl)-1H-pyrazol-4-yl)-5-(pyridin-4-yl)- 1,3,4-oxadiazol-3(2H)-yl)ethylidene)aniline: $5 \mathrm{~d}$}

IR $\left(\mathrm{KBr}, \mathrm{cm}^{-1}\right): 1590,1545\left(>\mathrm{C}=\mathrm{N}-,>\mathrm{C}=\mathrm{C}<\right.$ stretching), 1373 (-C-H stretching, $\left.-\mathrm{C}-\mathrm{CH}_{3}\right)$, $1291\left(>\mathrm{C}=\mathrm{N}\right.$ - stretching, Ar- $\left.\mathrm{NH}_{2}\right), 1232$ (C-O-C stretching, oxadiazole ring); ${ }^{1} \mathrm{H}$ NMR (DMSO-d $\mathrm{d}_{6}$, $400 \mathrm{MHz}): \delta=8.90\left(\mathrm{~d}, 2 \mathrm{H}, J=8.0 \mathrm{~Hz} \mathrm{C}_{2}-\underline{\mathrm{H}}\right.$ and $\mathrm{C}_{6}-\underline{\mathrm{H}}$ pyridine ring), $8.04\left(\mathrm{~d}, 2 \mathrm{H}, J=7.5 \mathrm{~Hz} \mathrm{C}_{3}-\underline{\mathrm{H}}\right.$ and $\mathrm{C}_{5}-\underline{\mathrm{H}}$ pyridine ring), $7.99\left(\mathrm{~s}, 1 \mathrm{H}, \mathrm{C}_{5}-\underline{\mathrm{H}}\right.$ pyrazole ring), 7.00-7.70 (m, 13H, Ar- $\left.\underline{\mathrm{H}}\right), 5.79(\mathrm{~s}, 1 \mathrm{H}$, $\mathrm{C}_{5}-\underline{\mathrm{H}}$ oxadiazole ring), $2.42\left(\mathrm{~s}, 6 \mathrm{H},-\underline{\mathrm{CH}}_{3}\right), 2.23\left(\mathrm{~s}, 3 \mathrm{H},-\mathrm{C}_{3}-\mathrm{C}=\mathrm{N}\right) ;{ }^{13} \mathrm{C}$ NMR $(100 \mathrm{MHz}, \mathrm{DMSO}-$ $\left.\mathrm{d}_{6}\right): \delta=157.4$ (1C, $\mathrm{C}_{5}$ oxadiazole ring), $156.3(1 \mathrm{C},-\mathrm{CH}=\mathrm{N}-), 149.8$ (2C, $\mathrm{C}_{2}$ and $\mathrm{C}_{6}$ pyridine ring), 149.2 (1C, $\mathrm{C}_{3}$ pyrazole ring), 139.1 (1C, $\mathrm{C}_{1}$ aromatic ring), 138.7 (1C, $\mathrm{C}_{4}$ pyridine ring), 136.7 (1C, $\left.\mathrm{C}_{2} \mathrm{C}_{5} \mathrm{H}_{4}-\mathrm{N}=\right)$, $131.7\left(1 \mathrm{C}, \mathrm{C}_{4} \mathrm{C}_{6} \mathrm{H}_{4}-\mathrm{CH}_{3}\right), 130.9\left(1 \mathrm{C}, \mathrm{C}_{3} \mathrm{C}_{5} \mathrm{H}_{4}-\mathrm{N}=\right)$, $130.6\left(1 \mathrm{C}, \mathrm{C}_{1} \mathrm{C}_{6} \mathrm{H}_{4}-\mathrm{CH}_{3}\right), 129.7$ (2C, $\mathrm{C}_{3}$ and $\left.\mathrm{C}_{5} \mathrm{C}_{6} \mathrm{H}_{4}-\mathrm{CH}_{3}\right), 129.4$ (2C, $\mathrm{C}_{3}$ and $\mathrm{C}_{5}$ aromatic ring), $128.1\left(1 \mathrm{C}, \mathrm{C}_{1} \mathrm{C}_{5} \mathrm{H}_{4}-\mathrm{N}=\right), 127.7$ $\left(1 \mathrm{C}, \mathrm{C}_{4} \mathrm{C}_{5} \mathrm{H}_{4}-\mathrm{N}=\right), 127.6\left(1 \mathrm{C}, \mathrm{C}_{5} \mathrm{C}_{5} \mathrm{H}_{4}-\mathrm{N}=\right), 126.8$ (1C, $\mathrm{C}_{4}$ aromatic ring), $125.3\left(2 \mathrm{C}, \mathrm{C}_{2}\right.$ and $\mathrm{C}_{6}$ $\left.\mathrm{C}_{5} \mathrm{H}_{4}-\mathrm{N}=\right), 124.5$ (2C, $\mathrm{C}_{3}$ and $\mathrm{C}_{5}$ pyridine ring), 123.8 (1C, $\mathrm{C}_{5}$ pyrazole ring), $120.6\left(1 \mathrm{C}, \mathrm{C}_{4} \mathrm{C}_{5} \mathrm{H}_{4}-\right.$ $\mathrm{N}=$ ), 119.4 (2C, $\mathrm{C}_{2}$ and $\mathrm{C}_{6}$ aromatic ring), 117.4 (1C, $\mathrm{C}_{4}$ pyrazole ring), 78.5 (1C, $\mathrm{C}_{2}$ oxadiazole ring), $21.4\left(1 \mathrm{C}, \mathrm{C}_{6} \mathrm{H}_{4}-\mathrm{CH}_{3}\right), 21.7\left(1 \mathrm{C},=\mathrm{CH}-\mathrm{CH}_{3}\right), 18.6\left(1 \mathrm{C}, \mathrm{CH}_{3}-\mathrm{C}_{5} \mathrm{H}_{4}-\mathrm{N}=\right)$; LCMS (ESI): m/z $513.2(37.2 \%)\left[\mathrm{M}^{+}\right]$; Anal. calcd. for $\mathrm{C}_{32} \mathrm{H}_{28} \mathrm{~N}_{6} \mathrm{O}: \mathrm{C}, 74.98 ; \mathrm{H}, 5.51 ; \mathrm{N}, 16.39$; Found: $\mathrm{C}, 75.03 ; \mathrm{H}$, $5.55 ; \mathrm{N}, 16.42 \%$. 


\section{Characterization of 3-methyl- $N$-(1-(2-(1-phenyl-3-(p-tolyl)-1H-pyrazol-4-yl)-5-(pyridin-4-yl)- 1,3,4-oxadiazol-3(2H)-yl)ethylidene)aniline: $5 \mathrm{e}$}

IR $\left(\mathrm{KBr}, \mathrm{cm}^{-1}\right):$ 1584, $1541\left(>\mathrm{C}=\mathrm{N}-,>\mathrm{C}=\mathrm{C}<\right.$ stretching), 1365 (-C-H stretching, $\left.-\mathrm{C}-\mathrm{CH}_{3}\right)$, $1285\left(>\mathrm{C}=\mathrm{N}\right.$ - stretching, Ar- $\left.\mathrm{NH}_{2}\right), 1229$ (C-O-C stretching, oxadiazole ring); ${ }^{1} \mathrm{H}$ NMR (DMSO-d $\mathrm{d}_{6}$,

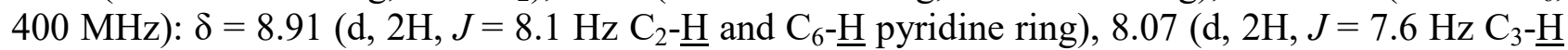
and $\mathrm{C}_{5}-\underline{\mathrm{H}}$ pyridine ring), $8.01\left(\mathrm{~s}, 1 \mathrm{H}, \mathrm{C}_{5}-\underline{\mathrm{H}}\right.$ pyrazole ring), $6.75-7.70(\mathrm{~m}, 13 \mathrm{H}, \mathrm{Ar}-\underline{\mathrm{H}}), 5.80(\mathrm{~s}, 1 \mathrm{H}$, $\mathrm{C}_{5}-\underline{\mathrm{H}}$ oxadiazole ring), $2.41\left(\mathrm{~s}, 6 \mathrm{H},-\mathrm{CH}_{3}\right), 2.24\left(\mathrm{~s}, 3 \mathrm{H},-\mathrm{C}_{3}-\mathrm{C}=\mathrm{N}\right) ;{ }^{13} \mathrm{C}$ NMR $(100 \mathrm{MHz}, \mathrm{DMSO}-$ $\left.\mathrm{d}_{6}\right): \delta=157.8\left(1 \mathrm{C}, \mathrm{C}_{5}\right.$ oxadiazole ring), $156.8(1 \mathrm{C},-\mathrm{CH}=\mathrm{N}-), 152.7\left(1 \mathrm{C}, \mathrm{C}_{1} \mathrm{C}_{5} \mathrm{H}_{4}-\mathrm{N}=\right), 149.7$ (1C, $\mathrm{C}_{3}$ pyrazole ring), $149.6\left(2 \mathrm{C}, \mathrm{C}_{2}\right.$ and $\mathrm{C}_{6}$ pyridine ring), $139.8(2 \mathrm{C}, 1 \mathrm{C}$ aromatic ring and $1 \mathrm{C}$ $\left.\mathrm{C}_{3} \mathrm{C}_{5} \mathrm{H}_{4}-\mathrm{N}=\right), 138.9$ (1C, $\mathrm{C}_{4}$ pyridine ring), $131.4\left(1 \mathrm{C}, \mathrm{C}_{4} \mathrm{C}_{6} \mathrm{H}_{4}-\mathrm{CH}_{3}\right), 130.9\left(1 \mathrm{C}, \mathrm{C}_{1} \mathrm{C}_{6} \mathrm{H}_{4}-\mathrm{CH}_{3}\right)$, $129.1\left(1 \mathrm{C}, \mathrm{C}_{5} \mathrm{C}_{5} \mathrm{H}_{4}-\mathrm{N}=\right)$, $129.6\left(2 \mathrm{C}, \mathrm{C}_{3}\right.$ and $\mathrm{C}_{5}$ aromatic ring), $129.6\left(2 \mathrm{C}, \mathrm{C}_{3}\right.$ and $\left.\mathrm{C}_{5} \mathrm{C}_{6} \mathrm{H}_{4}-\mathrm{CH}_{3}\right)$, $125.7\left(2 \mathrm{C}, \mathrm{C}_{2}\right.$ and $\left.\mathrm{C}_{6} \mathrm{C}_{6} \mathrm{H}_{4}-\mathrm{CH}_{3}\right), 124.6\left(2 \mathrm{C}, \mathrm{C}_{3}\right.$ and $\mathrm{C}_{5}$ pyridine ring), $123.1\left(1 \mathrm{C}, \mathrm{C}_{4} \mathrm{C}_{6} \mathrm{H}_{4}-\mathrm{CH}_{3}\right)$, 123.0 (1C, $\mathrm{C}_{5}$ pyrazole ring), $121.9\left(1 \mathrm{C}, \mathrm{C}_{2} \mathrm{C}_{5} \mathrm{H}_{4}-\mathrm{N}=\right), 119.5\left(2 \mathrm{C}, \mathrm{C}_{2}\right.$ and $\mathrm{C}_{6}$ aromatic ring), 119.7 $\left(1 \mathrm{C}, \mathrm{C}_{5} \mathrm{C}_{5} \mathrm{H}_{4}-\mathrm{N}=\right), 117.8$ (1C, $\mathrm{C}_{4}$ pyrazole ring), 78.2 (1C, $\mathrm{C}_{2}$ oxadiazole ring), 21.8 (2C, $\mathrm{CH}_{3}-\mathrm{C}_{5} \mathrm{H}_{4}-\mathrm{N}=$ and $\left.\mathrm{C}_{6} \mathrm{H}_{4}-\mathrm{CH}_{3}\right), 21.7\left(1 \mathrm{C},=\mathrm{CH}-\mathrm{CH}_{3}\right)$; LCMS (ESI): $\mathrm{m} / \mathrm{z} 513.2(37.2 \%)\left[\mathrm{M}^{+}\right.$]; Anal. calcd. for $\mathrm{C}_{32} \mathrm{H}_{28} \mathrm{~N}_{6} \mathrm{O}: \mathrm{C}, 74.98 ; \mathrm{H}, 5.51 ; \mathrm{N}, 16.39$; Found: $\mathrm{C}, 74.92 ; \mathrm{H}, 5.55 ; \mathrm{N}, 16.43 \%$.

\section{Characterization of 4-methyl- $\mathrm{N}$-(1-(2-(1-phenyl-3-(p-tolyl)-1H-pyrazol-4-yl)-5-(pyridin-4-yl)- 1,3,4-oxadiazol-3(2H)-yl)ethylidene)aniline: $5 \mathrm{f}$}

IR $\left(\mathrm{KBr}, \mathrm{cm}^{-1}\right): 1586,1538\left(>\mathrm{C}=\mathrm{N}-,>\mathrm{C}=\mathrm{C}<\right.$ stretching), 1369 (-C-H stretching, $\left.-\mathrm{C}-\mathrm{CH}_{3}\right)$, $1294\left(>\mathrm{C}=\mathrm{N}\right.$ - stretching, Ar- $\left.\mathrm{NH}_{2}\right), 1235$ (C-O-C stretching, oxadiazole ring); ${ }^{1} \mathrm{H}$ NMR (DMSO- $\mathrm{d}_{6}$, $400 \mathrm{MHz}): \delta=8.89\left(\mathrm{~d}, 2 \mathrm{H}, J=8.8 \mathrm{~Hz} \mathrm{C}_{2}-\underline{\mathrm{H}}\right.$ and $\mathrm{C}_{6}-\underline{\mathrm{H}}$ pyridine ring), $8.06\left(\mathrm{~d}, 2 \mathrm{H}, J=7.8 \mathrm{~Hz} \mathrm{C}_{3}-\underline{\mathrm{H}}\right.$ and $\mathrm{C}_{5}-\underline{\mathrm{H}}$ pyridine ring), $8.00\left(\mathrm{~s}, 1 \mathrm{H}, \mathrm{C}_{5}-\underline{\mathrm{H}}\right.$ pyrazole ring), 7.25-7.72 (m, 13H, Ar- $\left.\underline{\mathrm{H}}\right), 5.81(\mathrm{~s}, 1 \mathrm{H}$, $\mathrm{C}_{5}-\underline{\mathrm{H}}$ oxadiazole ring), $2.41\left(\mathrm{~s}, 6 \mathrm{H},-\mathrm{CH}_{3}\right), 2.22\left(\mathrm{~s}, 3 \mathrm{H},-\mathrm{C}_{3}-\mathrm{C}=\mathrm{N}\right) ;{ }^{13} \mathrm{C}$ NMR $(100 \mathrm{MHz}, \mathrm{DMSO}-$ $\left.\mathrm{d}_{6}\right): \delta=157.4$ (1C, $\mathrm{C}_{5}$ oxadiazole ring), $156.2(1 \mathrm{C},-\mathrm{CH}=\mathrm{N}-), 149.6$ (2C, $\mathrm{C}_{2}$ and $\mathrm{C}_{6}$ pyridine ring), 149.3 (1C, $\mathrm{C}_{3}$ pyrazole ring), $149.1\left(1 \mathrm{C}, \mathrm{C}_{1} \mathrm{C}_{5} \mathrm{H}_{4}-\mathrm{N}=\right)$, 139.3 (1C, $\mathrm{C}_{1}$ aromatic ring), 138.1 (1C, $\mathrm{C}_{4}$ pyridine ring), $136.4\left(1 \mathrm{C}, \mathrm{C}_{4} \mathrm{C}_{5} \mathrm{H}_{4}-\mathrm{N}=\right), 131.7\left(1 \mathrm{C}, \mathrm{C}_{4} \mathrm{C}_{6} \mathrm{H}_{4}-\mathrm{CH}_{3}\right), 130.9\left(1 \mathrm{C}, \mathrm{C}_{1} \mathrm{C}_{6} \mathrm{H}_{4}-\mathrm{CH}_{3}\right)$, $130.3\left(2 \mathrm{C}, \mathrm{C}_{3}\right.$ and $\left.\mathrm{C}_{5} \mathrm{C}_{5} \mathrm{H}_{4}-\mathrm{N}=\right), 129.7\left(2 \mathrm{C}, \mathrm{C}_{3}\right.$ and $\left.\mathrm{C}_{5} \mathrm{C}_{6} \mathrm{H}_{4}-\mathrm{CH}_{3}\right), 129.8\left(2 \mathrm{C}, \mathrm{C}_{3}\right.$ and $\mathrm{C}_{5}$ aromatic ring), 126.7 (1C, $\mathrm{C}_{1}$ aromatic ring), $125.8\left(2 \mathrm{C}, \mathrm{C}_{2}\right.$ and $\left.\mathrm{C}_{6} \mathrm{C}_{6} \mathrm{H}_{4}-\mathrm{CH}_{3}\right), 125.2\left(2 \mathrm{C}, \mathrm{C}_{2}\right.$ and $\mathrm{C}_{6}$ $\left.\mathrm{C}_{5} \mathrm{H}_{4}-\mathrm{N}=\right), 124.5$ (2C, $\mathrm{C}_{3}$ and $\mathrm{C}_{5}$ pyridine ring), 123.6 (1C, $\mathrm{C}_{5}$ pyrazole ring), $119.7\left(2 \mathrm{C}, \mathrm{C}_{2}\right.$ and $\mathrm{C}_{6}$ aromatic ring), $117.3\left(1 \mathrm{C}, \mathrm{C}_{4}\right.$ pyrazole ring), $78.0\left(1 \mathrm{C}, \mathrm{C}_{2}\right.$ oxadiazole ring), $21.4\left(2 \mathrm{C}_{1} \mathrm{CH}_{3}-\mathrm{C}_{5} \mathrm{H}_{4}-\mathrm{N}=\right.$ and $\left.\mathrm{C}_{6} \mathrm{H}_{4}-\mathrm{CH}_{3}\right), 21.6\left(1 \mathrm{C},=\mathrm{CH}-\mathrm{CH}_{3}\right) ; \mathrm{LCMS}(\mathrm{ESI}): \mathrm{m} / \mathrm{z} 513.2(37.2 \%)\left[\mathrm{M}^{+}\right]$; Anal. calcd. for $\mathrm{C}_{32} \mathrm{H}_{28} \mathrm{~N}_{6} \mathrm{O}$ : C, 74.98; H, 5.51; N, 16.39; Found: C, 75.06; H, 5.56; N, 16.41\%.

Characterization of 2-methoxy- $N$-(1-(2-(1-phenyl-3-(p-tolyl)-1H-pyrazol-4-yl)-5-(pyridin-4yl)-1,3,4-oxadiazol-3(2H)-yl)ethylidene)aniline: $5 \mathrm{~g}$

IR $\left(\mathrm{KBr}, \mathrm{cm}^{-1}\right): 2830\left(\mathrm{C}-\mathrm{H}\right.$ stretching, $\left.-\mathrm{OCH}_{3}\right), 1580,1542(>\mathrm{C}=\mathrm{N}-,>\mathrm{C}=\mathrm{C}<$ stretching $), 1371$ (-C-H stretching, -C- $\left.\mathrm{CH}_{3}\right), 1290\left(>\mathrm{C}=\mathrm{N}\right.$ - stretching, Ar- $\left.\mathrm{NH}_{2}\right), 1232$ (C-O-C stretching, oxadiazole

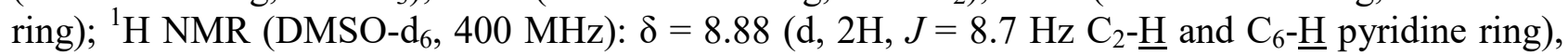
$8.02\left(\mathrm{~s}, 1 \mathrm{H}, \mathrm{C}_{5}-\underline{\mathrm{H}}\right.$ pyrazole ring), $8.08\left(\mathrm{~d}, 2 \mathrm{H}, J=7.5 \mathrm{~Hz} \mathrm{C}_{3}-\underline{\mathrm{H}}\right.$ and $\mathrm{C}_{5}-\underline{\mathrm{H}}$ pyridine ring), 6.70-7.67 $(\mathrm{m}, 13 \mathrm{H}, A r-\underline{H}), 5.82\left(\mathrm{~s}, 1 \mathrm{H}, \mathrm{C}_{5}-\underline{\mathrm{H}}\right.$ oxadiazole ring), $3.89\left(\mathrm{~s}, 3 \mathrm{H},-\mathrm{OC} \underline{\mathrm{H}}_{3}\right), 2.39\left(\mathrm{~s}, 3 \mathrm{H},-\underline{\mathrm{H}}_{3}\right)$, $2.24\left(\mathrm{~s}, 3 \mathrm{H},-\mathrm{C}_{3}-\mathrm{C}=\mathrm{N}\right) ;{ }^{13} \mathrm{C} \mathrm{NMR}\left(100 \mathrm{MHz}, \mathrm{DMSO}-\mathrm{d}_{6}\right): \delta=157.8\left(1 \mathrm{C}, \mathrm{C}_{5}\right.$ oxadiazole ring), $156.0(1 \mathrm{C},-\mathrm{CH}=\mathrm{N}-), 152.1\left(1 \mathrm{C}, \mathrm{C}_{2} \mathrm{C}_{5} \mathrm{H}_{4}-\mathrm{N}=\right), 149.4$ (1C, $\mathrm{C}_{3}$ pyrazole ring), $149.2\left(2 \mathrm{C}, \mathrm{C}_{2}\right.$ and $\mathrm{C}_{6}$ pyridine ring), 139.1 ( $1 \mathrm{C}, \mathrm{C}_{1}$ aromatic ring), 138.0 (1C, $\mathrm{C}_{4}$ pyridine ring), $135.6\left(1 \mathrm{C}, \mathrm{C}_{1} \mathrm{C}_{5} \mathrm{H}_{4}-\mathrm{N}=\right)$, $131.2\left(1 \mathrm{C}, \mathrm{C}_{4} \mathrm{C}_{6} \mathrm{H}_{4}-\mathrm{CH}_{3}\right), 130.4\left(1 \mathrm{C}, \mathrm{C}_{1} \mathrm{C}_{6} \mathrm{H}_{4}-\mathrm{CH}_{3}\right), 129.8\left(2 \mathrm{C}, \mathrm{C}_{3}\right.$ and $\mathrm{C}_{5}$ aromatic ring), 129.6 (2C, $\mathrm{C}_{3}$ and $\left.\mathrm{C}_{5} \mathrm{C}_{6} \mathrm{H}_{4}-\mathrm{CH}_{3}\right), 126.5$ (1C, $\mathrm{C}_{4}$ aromatic ring), $125.4\left(2 \mathrm{C}, \mathrm{C}_{2}\right.$ and $\mathrm{C}_{6} \mathrm{C}_{6} \mathrm{H}_{4}-\mathrm{CH}_{3}$ ), 124.5 (2C, $\mathrm{C}_{3}$ and $\mathrm{C}_{5}$ pyridine ring), $124.2\left(1 \mathrm{C}, \mathrm{C}_{4} \mathrm{C}_{5} \mathrm{H}_{4}-\mathrm{N}=\right), 123.7$ (1C, $\mathrm{C}_{5}$ pyrazole ring), 122.0 (1C, $\left.\mathrm{C}_{5} \mathrm{C}_{5} \mathrm{H}_{4}-\mathrm{N}=\right), 119.3$ (2C, $\mathrm{C}_{2}$ and $\mathrm{C}_{6}$ aromatic ring), 117.4 (1C, $\mathrm{C}_{4}$ pyrazole ring), 115.6 (1C, $\left.\mathrm{C}_{3} \mathrm{C}_{5} \mathrm{H}_{4}-\mathrm{N}=\right)$, $115.1\left(1 \mathrm{C}, \mathrm{C}_{3} \mathrm{C}_{5} \mathrm{H}_{4}-\mathrm{N}=\right)$, 78.5 (1C, $\mathrm{C}_{2}$ oxadiazole ring), $55.4\left(1 \mathrm{C},-\mathrm{OCH}_{3}\right), 21.4(1 \mathrm{C}$, $\left.\mathrm{C}_{6} \mathrm{H}_{4}-\mathrm{CH}_{3}\right), 21.0\left(1 \mathrm{C},=\mathrm{CH}-\mathrm{CH}_{3}\right)$; LCMS (ESI): $\mathrm{m} / \mathrm{z} 528.2(100.0 \%)\left[\mathrm{M}^{+}\right]$, Anal. calcd. for $\mathrm{C}_{32} \mathrm{H}_{28} \mathrm{~N}_{6} \mathrm{O}_{2}$ : C, 72.71; H, 5.34; N, 15.90; Found: C, 72.75; H, 5.39; N, 15.95\%. 
Characterization of 3-methoxy- $N$-(1-(2-(1-phenyl-3-(p-tolyl)-1H-pyrazol-4-yl)-5-(pyridin-4yl)-1,3,4-oxadiazol-3(2H)-yl)ethylidene)aniline: $5 \mathrm{~h}$

IR $\left(\mathrm{KBr}, \mathrm{cm}^{-1}\right)$ : $2825\left(\mathrm{C}-\mathrm{H}\right.$ stretching, $\left.-\mathrm{OCH}_{3}\right), 1587,1543(>\mathrm{C}=\mathrm{N}-,>\mathrm{C}=\mathrm{C}<$ stretching), 1368 (-C-H stretching, $\left.-\mathrm{C}-\mathrm{CH}_{3}\right), 1288\left(>\mathrm{C}=\mathrm{N}\right.$ - stretching, Ar- $\left.\mathrm{NH}_{2}\right), 1237$ (C-O-C stretching, oxadiazole

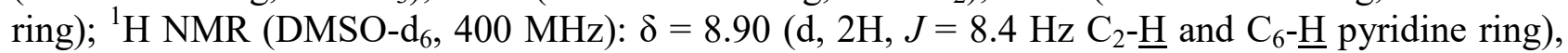
$8.10\left(\mathrm{~d}, 2 \mathrm{H}, J=7.7 \mathrm{~Hz} \mathrm{C}_{3}-\underline{\mathrm{H}}\right.$ and $\mathrm{C}_{5}-\underline{\mathrm{H}}$ pyridine ring), 8.01 (s, $1 \mathrm{H}, \mathrm{C}_{5}-\underline{\mathrm{H}}$ pyrazole ring), 6.60-7.60 (m, 13H, Ar- $\underline{\mathrm{H}}), 5.83\left(\mathrm{~s}, 1 \mathrm{H}, \mathrm{C}_{5}-\underline{\mathrm{H}}\right.$ oxadiazole ring), $3.88\left(\mathrm{~s}, 3 \mathrm{H},-\mathrm{OC} \underline{\mathrm{H}}_{3}\right), 2.39\left(\mathrm{~s}, 3 \mathrm{H},-\mathrm{C}_{3}\right), 2.26$ $\left(\mathrm{s}, 3 \mathrm{H},-\mathrm{CH}_{3}-\mathrm{C}=\mathrm{N}\right) ;{ }^{13} \mathrm{C}$ NMR $\left(100 \mathrm{MHz}, \mathrm{DMSO}-\mathrm{d}_{6}\right): \delta=161.4\left(1 \mathrm{C}, \overline{\mathrm{C}}_{3} \mathrm{C}_{6} \mathrm{H}_{4}-\mathrm{CH}_{3}\right), 157.5\left(1 \mathrm{C}, \mathrm{C}_{5}\right.$ oxadiazole ring), $156.1(1 \mathrm{C},-\mathrm{CH}=\mathrm{N}-), 150.6\left(1 \mathrm{C}, \mathrm{C}_{1} \mathrm{C}_{5} \mathrm{H}_{4}-\mathrm{N}=\right), 149.3\left(1 \mathrm{C}, \mathrm{C}_{3}\right.$ pyrazole ring), 149.0 (2C, $\mathrm{C}_{2}$ and $\mathrm{C}_{6}$ pyridine ring), 139.5 (1C, $\mathrm{C}_{1}$ aromatic ring), 138.1 (1C, $\mathrm{C}_{4}$ aromatic ring), 131.7 $\left(1 \mathrm{C}, \mathrm{C}_{4} \mathrm{C}_{6} \mathrm{H}_{4}-\mathrm{CH}_{3}\right), 131.3\left(1 \mathrm{C}, \mathrm{C}_{4} \mathrm{C}_{6} \mathrm{H}_{4}-\mathrm{CH}_{3}\right), 130.3\left(1 \mathrm{C}, \mathrm{C}_{1} \mathrm{C}_{6} \mathrm{H}_{4}-\mathrm{CH}_{3}\right), 129.7\left(2 \mathrm{C}, \mathrm{C}_{3}\right.$ and $\mathrm{C}_{5}$ aromatic ring), 129.3 (2C, $\mathrm{C}_{3}$ and $\left.\mathrm{C}_{5} \mathrm{C}_{6} \mathrm{H}_{4}-\mathrm{CH}_{3}\right), 126.5$ (1C, $\mathrm{C}_{4}$ aromatic ring), $125.3\left(2 \mathrm{C}, \mathrm{C}_{2}\right.$ and $\mathrm{C}_{6}$ $\left.\mathrm{C}_{6} \mathrm{H}_{4}-\mathrm{CH}_{3}\right), 124.7$ (2C, $\mathrm{C}_{3}$ and $\mathrm{C}_{5}$ pyridine ring), 123.7 (1C, $\mathrm{C}_{5}$ pyrazole ring), 119.5 (2C, $\mathrm{C}_{2}$ and $\mathrm{C}_{6}$ aromatic ring), $117.6\left(1 \mathrm{C}, \mathrm{C}_{4}\right.$ pyrazole ring), $114.9\left(1 \mathrm{C}, \mathrm{C}_{1} \mathrm{C}_{5} \mathrm{H}_{4}-\mathrm{N}=\right), 108.5\left(1 \mathrm{C}, \mathrm{C}_{2} \mathrm{C}_{5} \mathrm{H}_{4}-\mathrm{N}=\right)$, $106.7\left(1 \mathrm{C}, \mathrm{C}_{4} \mathrm{C}_{5} \mathrm{H}_{4}-\mathrm{N}=\right)$, $78.3\left(1 \mathrm{C}, \mathrm{C}_{2}\right.$ oxadiazole ring), $55.3\left(1 \mathrm{C},-\mathrm{OCH}_{3}\right), 21.4\left(1 \mathrm{C}, \mathrm{C}_{6} \mathrm{H}_{4}-\mathrm{CH}_{3}\right)$, $21.0\left(1 \mathrm{C},=\mathrm{CH}-\mathrm{CH}_{3}\right)$; LCMS (ESI): $\mathrm{m} / \mathrm{z} 528.2(100.0 \%)\left[\mathrm{M}^{+}\right]$; Anal. calcd. for $\mathrm{C}_{32} \mathrm{H}_{28} \mathrm{~N}_{6} \mathrm{O}_{2}$ : C, 72.71; H, 5.34; N, 15.90; Found: C, 72.76; H, 5.40; N, 15.95\%.

Characterization of 4-methoxy- $\mathrm{N}$-(1-(2-(1-phenyl-3-(p-tolyl)-1H-pyrazol-4-yl)-5-(pyridin-4yl)-1,3,4-oxadiazol-3(2H)-yl)ethylidene)aniline: $5 \mathrm{i}$

IR $\left(\mathrm{KBr}, \mathrm{cm}^{-1}\right): 2820\left(\mathrm{C}-\mathrm{H}\right.$ stretching, $\left.-\mathrm{OCH}_{3}\right), 1581,1536(>\mathrm{C}=\mathrm{N}-,>\mathrm{C}=\mathrm{C}<$ stretching $), 1364$ (-C-H stretching, $\left.-\mathrm{C}-\mathrm{CH}_{3}\right), 1283\left(>\mathrm{C}=\mathrm{N}\right.$ - stretching, Ar- $\left.\mathrm{NH}_{2}\right), 1235(\mathrm{C}-\mathrm{O}-\mathrm{C}$ stretching, oxadiazole

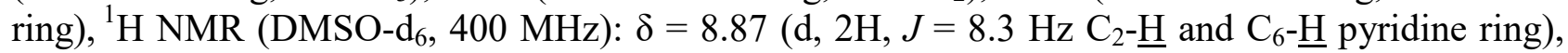
8.07 (d, $2 \mathrm{H}, J=7.7 \mathrm{~Hz} \mathrm{C}_{3}-\underline{\mathrm{H}}$ and $\mathrm{C}_{5}-\underline{\mathrm{H}}$ pyridine ring), 8.03 (s, $1 \mathrm{H}, \mathrm{C}_{5}-\underline{\mathrm{H}}$ pyrazole ring), 7.10-7.67 $(\mathrm{m}, 13 \mathrm{H}, \mathrm{Ar}-\underline{\mathrm{H}}), 5.86\left(\mathrm{~s}, 1 \mathrm{H}, \mathrm{C}_{5}-\underline{\mathrm{H}}\right.$ oxadiazole ring), $3.90\left(\mathrm{~s}, 3 \mathrm{H},-\mathrm{OC}^{\mathrm{H}} 3\right), 2.37\left(\mathrm{~s}, 3 \mathrm{H},-\underline{\mathrm{C}}_{3}\right), 2.27$ $\left(\mathrm{s}, 3 \mathrm{H},-\mathrm{CH}_{3}-\mathrm{C}=\mathrm{N}\right) ;{ }^{13} \mathrm{C}$ NMR $\left(100 \mathrm{MHz}, \mathrm{DMSO}-\mathrm{d}_{6}\right): \delta=159.2\left(1 \mathrm{C}, \overline{\mathrm{C}}_{4} \mathrm{C}_{6} \mathrm{H}_{4}-\mathrm{CH}_{3}\right), 157.6\left(1 \mathrm{C}, \mathrm{C}_{5}\right.$ oxadiazole ring), $156.1(1 \mathrm{C},-\mathrm{CH}=\mathrm{N}-), 149.8$ (1C, $\mathrm{C}_{3}$ pyrazole ring), $149.3\left(2 \mathrm{C}_{1} \mathrm{C}_{2}\right.$ and $\mathrm{C}_{6}$ pyridine ring), $144.3\left(1 \mathrm{C}, \mathrm{C}_{1} \mathrm{C}_{5} \mathrm{H}_{4}-\mathrm{N}=\right), 139.1$ (1C, $\mathrm{C}_{1}$ aromatic ring), 138.7 (1C, $\mathrm{C}_{4}$ pyridine ring), 131.3 (1C, $\left.\mathrm{C}_{4} \mathrm{C}_{6} \mathrm{H}_{4}-\mathrm{CH}_{3}\right), 130.4\left(1 \mathrm{C}, \mathrm{C}_{1} \mathrm{C}_{6} \mathrm{H}_{4}-\mathrm{CH}_{3}\right), 129.1$ (2C, $\mathrm{C}_{3}$ and $\mathrm{C}_{5}$ aromatic ring), 129.1 (2C, $\mathrm{C}_{3}$ and $\left.\mathrm{C}_{5} \mathrm{C}_{6} \mathrm{H}_{4}-\mathrm{CH}_{3}\right), 126.7$ (1C, $\mathrm{C}_{4}$ aromatic ring), $125.8\left(2 \mathrm{C}, \mathrm{C}_{2}\right.$ and $\left.\mathrm{C}_{6} \mathrm{C}_{6} \mathrm{H}_{4}-\mathrm{CH}_{3}\right), 124.6\left(2 \mathrm{C}, \mathrm{C}_{3}\right.$ and $\mathrm{C}_{5}$ pyridine ring), 123.2 (1C, $\mathrm{C}_{5}$ pyrazole ring), 122.7 (2C, $\mathrm{C}_{2}$ and $\left.\mathrm{C}_{6} \mathrm{C}_{6} \mathrm{H}_{4}-\mathrm{CH}_{3}\right), 119.5\left(2 \mathrm{C}, \mathrm{C}_{2}\right.$ and $\mathrm{C}_{6}$ aromatic ring), 117.5 (1C, $\mathrm{C}_{4}$ pyrazole ring), $115.7\left(2 \mathrm{C}, \mathrm{C}_{3}\right.$ and $\left.\mathrm{C}_{5} \mathrm{C}_{5} \mathrm{H}_{4}-\mathrm{N}=\right), 78.1\left(1 \mathrm{C}, \mathrm{C}_{2}\right.$ oxadiazole ring), $55.1\left(1 \mathrm{C},-\mathrm{OCH}_{3}\right), 21.4\left(1 \mathrm{C}, \mathrm{C}_{6} \mathrm{H}_{4}-\mathrm{CH}_{3}\right), 21.3\left(1 \mathrm{C},=\mathrm{CH}-\mathrm{CH}_{3}\right) ; \mathrm{LCMS}(\mathrm{ESI}): \mathrm{m} / \mathrm{z}$ $528.2(100.0 \%)\left[\mathrm{M}^{+}\right]$; Anal. calcd. for $\mathrm{C}_{32} \mathrm{H}_{28} \mathrm{~N}_{6} \mathrm{O}_{2}$ : C, 72.71; H, 5.34; N, 15.90; Found: C, 72.75; $\mathrm{H}, 5.42 ; \mathrm{N}, 15.96 \%$.

Characterization of 2-nitro- $N$-(1-(2-(1-phenyl-3-(p-tolyl)-1 $H$-pyrazol-4-yl)-5-(pyridin-4-yl)1,3,4-oxadiazol-3(2H)-yl)ethylidene)aniline: 5 j

IR $\left(\mathrm{KBr}, \mathrm{cm}^{-1}\right): 1585,1540(>\mathrm{C}=\mathrm{N}-,>\mathrm{C}=\mathrm{C}<$ stretching $), 1510\left(-\mathrm{N}=\mathrm{O}\right.$ stretching, Ar- $\left.\mathrm{NO}_{2}\right)$, 1370(-C-H stretching, -C- $\left.\mathrm{CH}_{3}\right), 1298\left(>\mathrm{C}=\mathrm{N}\right.$ - stretching, Ar- $\left.\mathrm{NH}_{2}\right), 1232(\mathrm{C}-\mathrm{O}-\mathrm{C}$ stretching, oxadiazole ring); ${ }^{1} \mathrm{H}$ NMR (DMSO-d $\left.{ }_{6}, 400 \mathrm{MHz}\right): \delta=8.85\left(\mathrm{~d}, 2 \mathrm{H}, J=8.3 \mathrm{~Hz} \mathrm{C}_{2}-\underline{\mathrm{H}}\right.$ and $\mathrm{C}_{6}-\underline{\mathrm{H}}$

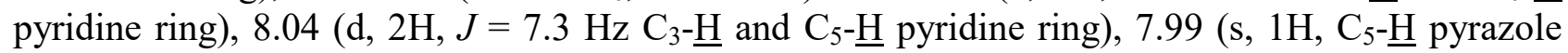
ring), 7.60-8.07 (m, 13H, Ar- $\underline{\mathrm{H}}), 5.88\left(\mathrm{~s}, 1 \mathrm{H}, \mathrm{C}_{5}-\underline{\mathrm{H}}\right.$ oxadiazole ring), $2.35\left(\mathrm{~s}, 3 \mathrm{H},-\underline{\mathrm{C}}_{3}\right), 2.25(\mathrm{~s}, 3 \mathrm{H}$, $\left.-\underline{\mathrm{H}}_{3}-\mathrm{C}=\mathrm{N}\right) ;{ }^{13} \mathrm{C}$ NMR $\left(100 \mathrm{MHz}, \mathrm{DMSO}-\mathrm{d}_{6}\right): \bar{\delta}=157.4$ (1C, $\mathrm{C}_{5}$ oxadiazole ring), 156.1 (1C, $\mathrm{CH}=\mathrm{N}-), 149.5$ (1C, $\mathrm{C}_{3}$ pyrazole ring), 149.3 (2C, $\mathrm{C}_{2}$ and $\mathrm{C}_{6}$ pyridine ring), $145.7\left(1 \mathrm{C}, \mathrm{C}_{2} \mathrm{C}_{5} \mathrm{H}_{4}-\right.$ $\mathrm{N}=), 142.3\left(1 \mathrm{C}, \mathrm{C}_{2} \mathrm{C}_{5} \mathrm{H}_{4}-\mathrm{N}=\right), 139.1$ (1C, $\mathrm{C}_{1}$ aromatic ring), 138.2 (1C, $\mathrm{C}_{4}$ pyridine ring), 131.4 $\left(1 \mathrm{C}, \mathrm{C}_{4} \mathrm{C}_{5} \mathrm{H}_{4}-\mathrm{N}=\right)$, $131.1\left(1 \mathrm{C}, \mathrm{C}_{4} \mathrm{C}_{6} \mathrm{H}_{4}-\mathrm{CH}_{3}\right), 130.5\left(2 \mathrm{C}, \mathrm{C}_{6} \mathrm{C}_{5} \mathrm{H}_{4}-\mathrm{N}=\right.$ and $\left.\mathrm{C}_{4} \mathrm{C}_{6} \mathrm{H}_{4}-\mathrm{CH}_{3}\right), 129.7$ (2C, $\mathrm{C}_{3}$ and $\left.\mathrm{C}_{5} \mathrm{C}_{6} \mathrm{H}_{4}-\mathrm{CH}_{3}\right), 129.4\left(2 \mathrm{C}, \mathrm{C}_{3}\right.$ and $\mathrm{C}_{5}$ aromatic ring), $128.6\left(1 \mathrm{C}, \mathrm{C}_{4} \mathrm{C}_{5} \mathrm{H}_{4}-\mathrm{N}=\right), 126.7$ (1C, $\mathrm{C}_{4}$ aromatic ring), $125.7\left(2 \mathrm{C}, \mathrm{C}_{2}\right.$ and $\left.\mathrm{C}_{6} \mathrm{C}_{6} \mathrm{H}_{4}-\mathrm{CH}_{3}\right), 125.6\left(1 \mathrm{C}, \mathrm{C}_{3} \mathrm{C}_{5} \mathrm{H}_{4}-\mathrm{N}=\right), 124.6\left(2 \mathrm{C}, \mathrm{C}_{3}\right.$ and $\mathrm{C}_{5}$ pyridine ring), 123.8 (1C, $\mathrm{C}_{5}$ pyrazole ring), 119.7 (2C, $\mathrm{C}_{2}$ and $\mathrm{C}_{6}$ aromatic ring), 117.2 (1C, $\mathrm{C}_{4}$ pyrazole ring), 78.5 (1C, $\mathrm{C}_{2}$ oxadiazole ring), $21.4\left(1 \mathrm{C}, \mathrm{C}_{6} \mathrm{H}_{4}-\mathrm{CH}_{3}\right), 21.0\left(1 \mathrm{C},=\mathrm{CH}-\mathrm{CH}_{3}\right)$; 
LCMS (ESI): m/z 544.2 (36.5\%) $\left[\mathrm{M}^{+}\right]$; Anal. calcd. for $\mathrm{C}_{31} \mathrm{H}_{25} \mathrm{~N}_{7} \mathrm{O}_{3}: \mathrm{C}, 68.50 ; \mathrm{H}, 4.64 ; \mathrm{N}, 18.04$; Found: C, 68.55; H, 4.69; N, 18.10\%.

\section{Characterization of 3-nitro- $N$-(1-(2-(1-phenyl-3-(p-tolyl)-1 $H$-pyrazol-4-yl)-5-(pyridin-4-yl)- 1,3,4-oxadiazol-3(2H)-yl)ethylidene)aniline: $5 \mathrm{k}$}

IR $\left(\mathrm{KBr}, \mathrm{cm}^{-1}\right): 1590,1532(>\mathrm{C}=\mathrm{N}-,>\mathrm{C}=\mathrm{C}<$ stretching $), 1500\left(-\mathrm{N}=\mathrm{O}\right.$ stretching, Ar- $\left.\mathrm{NO}_{2}\right)$, 1365 (-C-H stretching, -C- $\left.\mathrm{CH}_{3}\right), 1295\left(>\mathrm{C}=\mathrm{N}\right.$ - stretching, Ar- $\left.\mathrm{NH}_{2}\right), 1228(\mathrm{C}-\mathrm{O}-\mathrm{C}$ stretching, oxadiazole ring); ${ }^{1} \mathrm{H}$ NMR (DMSO- $\left.\mathrm{d}_{6}, 400 \mathrm{MHz}\right): \delta=8.84\left(\mathrm{~d}, 2 \mathrm{H}, J=8.3 \mathrm{~Hz} \mathrm{C}_{2}-\underline{\mathrm{H}}\right.$ and $\mathrm{C}_{6}-\underline{\mathrm{H}}$ pyridine ring), $8.09\left(\mathrm{~d}, 2 \mathrm{H}, J=7.3 \mathrm{~Hz} \mathrm{C}_{3}-\underline{\mathrm{H}}\right.$ and $\mathrm{C}_{5}-\underline{\mathrm{H}}$ pyridine ring), 7.97 (s, $1 \mathrm{H}, \mathrm{C}_{5}-\underline{\mathrm{H}}$ pyrazole ring), 7.35-7.80 (m, 13H, Ar- $\underline{\mathrm{H}}), 5.89\left(\mathrm{~s}, 1 \mathrm{H}, \mathrm{C}_{5}-\underline{\mathrm{H}}\right.$ oxadiazole ring), $2.34\left(\mathrm{~s}, 3 \mathrm{H},-\mathrm{C}_{3}\right), 2.26(\mathrm{~s}, 3 \mathrm{H}$, $\left.-\underline{\mathrm{H}}_{3}-\mathrm{C}=\mathrm{N}\right) ;{ }^{13} \mathrm{C}$ NMR $\left(100 \mathrm{MHz}, \mathrm{DMSO}-\mathrm{d}_{6}\right): \delta=157.4\left(1 \mathrm{C}, \mathrm{C}_{5}\right.$ oxadiazole ring), 156.2 (1C, $-\mathrm{CH}=\mathrm{N}-), 149.4\left(2 \mathrm{C}, \mathrm{C}_{3}\right.$ pyrazole ring and $\left.\mathrm{C}_{1} \mathrm{C}_{5} \mathrm{H}_{4}-\mathrm{N}=\right), 149.2\left(1 \mathrm{C}, \mathrm{C}_{3} \mathrm{C}_{5} \mathrm{H}_{4}-\mathrm{N}=\right), 149.0\left(2 \mathrm{C}, \mathrm{C}_{2}\right.$ and $\mathrm{C}_{6}$ pyridine ring), $139.1\left(1 \mathrm{C}, \mathrm{C}_{1}\right.$ aromatic ring), $138.0\left(1 \mathrm{C}, \mathrm{C}_{4}\right.$ pyridine ring), $132.7\left(1 \mathrm{C}, \mathrm{C}_{5}\right.$ $\left.\mathrm{C}_{5} \mathrm{H}_{4}-\mathrm{N}=\right), 131.2\left(1 \mathrm{C}, \mathrm{C}_{4} \mathrm{C}_{6} \mathrm{H}_{4}-\mathrm{CH}_{3}\right), 130.8\left(1 \mathrm{C}, \mathrm{C}_{1} \mathrm{C}_{6} \mathrm{H}_{4}-\mathrm{CH}_{3}\right), 129.4\left(2 \mathrm{C}, \mathrm{C}_{3}\right.$ and $\mathrm{C}_{5}$ aromatic ring), $129.0\left(2 \mathrm{C}, \mathrm{C}_{3}\right.$ and $\left.\mathrm{C}_{5} \mathrm{C}_{6} \mathrm{H}_{4}-\mathrm{CH}_{3}\right), 128.6\left(1 \mathrm{C}, \mathrm{C}_{6} \mathrm{C}_{5} \mathrm{H}_{4}-\mathrm{N}=\right)$, 126.7 (1C, $\mathrm{C}_{4}$ aromatic ring), 125.1 (2C, $\mathrm{C}_{2}$ and $\left.\mathrm{C}_{6} \mathrm{C}_{6} \mathrm{H}_{4}-\mathrm{CH}_{3}\right), 124.8$ (2C, $\mathrm{C}_{3}$ and $\mathrm{C}_{5}$ pyridine ring), 123.0 (1C, $\mathrm{C}_{5}$ pyrazole ring), $122.7\left(1 \mathrm{C}, \mathrm{C}_{4} \mathrm{C}_{5} \mathrm{H}_{4}-\mathrm{N}=\right), 119.5\left(2 \mathrm{C}, \mathrm{C}_{2}\right.$ and $\mathrm{C}_{6}$ aromatic ring), $118.3\left(1 \mathrm{C}, \mathrm{C}_{2} \mathrm{C}_{5} \mathrm{H}_{4}-\mathrm{N}=\right), 117.7(1 \mathrm{C}$, $\mathrm{C}_{4}$ pyrazole ring), 78.9 (1C, $\mathrm{C}_{2}$ oxadiazole ring), $21.5\left(1 \mathrm{C}, \mathrm{C}_{6} \mathrm{H}_{4}-\mathrm{CH}_{3}\right), 21.3\left(1 \mathrm{C},=\mathrm{CH}-\mathrm{CH}_{3}\right)$; LCMS (ESI): $\mathrm{m} / \mathrm{z} 544.2(36.5 \%)\left[\mathrm{M}^{+}\right]$; Anal. calcd. for $\mathrm{C}_{31} \mathrm{H}_{25} \mathrm{~N}_{7} \mathrm{O}_{3}: \mathrm{C}, 68.50 ; \mathrm{H}, 4.64 ; \mathrm{N}, 18.04$; Found: C, 68.56; H, 4.69; N, 18.10\%.

Characterization of 4-nitro- $N$-(1-(2-(1-phenyl-3-(p-tolyl)-1H-pyrazol-4-yl)-5-(pyridin-4-yl)1,3,4-oxadiazol-3(2H)-yl)ethylidene)aniline: $5 \mathrm{l}$

IR $\left(\mathrm{KBr}, \mathrm{cm}^{-1}\right):$ 1597, $1537(>\mathrm{C}=\mathrm{N}-,>\mathrm{C}=\mathrm{C}<$ stretching $), 1504\left(-\mathrm{N}=\mathrm{O}\right.$ stretching, Ar- $\left.\mathrm{NO}_{2}\right)$, 1355 (-C-H stretching, -C- $\left.\mathrm{CH}_{3}\right), 1292\left(>\mathrm{C}=\mathrm{N}\right.$ - stretching, Ar- $\left.\mathrm{NH}_{2}\right), 1224(\mathrm{C}-\mathrm{O}-\mathrm{C}$ stretching,

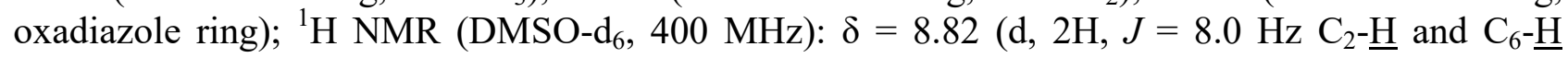

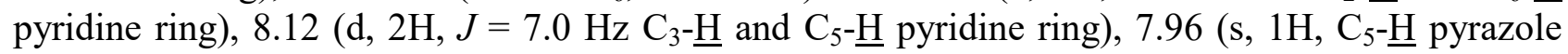
ring), 6.95-8.15 (m, 13H, Ar- $\underline{\mathrm{H}}), 5.85\left(\mathrm{~s}, 1 \mathrm{H}, \mathrm{C}_{5}-\underline{\mathrm{H}}\right.$ oxadiazole ring), $2.39\left(\mathrm{~s}, 3 \mathrm{H},-\underline{\mathrm{C}}_{3}\right), 2.28(\mathrm{~s}, 3 \mathrm{H}$, $\left.-\underline{\mathrm{H}}_{3}-\mathrm{C}=\mathrm{N}\right) ;{ }^{13} \mathrm{C}$ NMR $\left(100 \mathrm{MHz}, \mathrm{DMSO}-\mathrm{d}_{6}\right): \delta=158.3\left(1 \mathrm{C}, \mathrm{C}_{1} \mathrm{C}_{5} \mathrm{H}_{4}-\mathrm{N}=\right), 157.0\left(1 \mathrm{C}, \mathrm{C}_{5}\right.$ oxadiazole ring), $156.3(1 \mathrm{C},-\mathrm{CH}=\mathrm{N}-), 149.5\left(1 \mathrm{C}, \mathrm{C}_{3}\right.$ pyrazole ring $), 149.2\left(2 \mathrm{C}, \mathrm{C}_{2}\right.$ and $\mathrm{C}_{6}$ pyridine ring), $146.2\left(1 \mathrm{C}, \mathrm{C}_{4} \mathrm{C}_{5} \mathrm{H}_{4}-\mathrm{N}=\right), 139.1$ (1C, $\mathrm{C}_{1}$ aromatic ring), 138.2 (1C, $\mathrm{C}_{4}$ pyridine ring), 131.0 $\left(1 \mathrm{C}, \mathrm{C}_{4} \mathrm{C}_{6} \mathrm{H}_{4}-\mathrm{CH}_{3}\right), 130.5\left(1 \mathrm{C}, \mathrm{C}_{1} \mathrm{C}_{6} \mathrm{H}_{4}-\mathrm{CH}_{3}\right), 129.4\left(2 \mathrm{C}, \mathrm{C}_{3}\right.$ and $\left.\mathrm{C}_{5} \mathrm{C}_{6} \mathrm{H}_{4}-\mathrm{CH}_{3}\right), 129.0\left(2 \mathrm{C}, \mathrm{C}_{3}\right.$ and $\mathrm{C}_{5}$ aromatic ring), $128.6\left(1 \mathrm{C}, \mathrm{C}_{6} \mathrm{C}_{5} \mathrm{H}_{4}-\mathrm{N}=\right), 126.2$ (1C, $\mathrm{C}_{4}$ aromatic ring), $125.5\left(2 \mathrm{C}, \mathrm{C}_{2}\right.$ and $\mathrm{C}_{6}$ $\left.\mathrm{C}_{6} \mathrm{H}_{4}-\mathrm{CH}_{3}\right), 125.2\left(2 \mathrm{C}, \mathrm{C}_{3} \mathrm{C}_{5} \mathrm{H}_{4}-\mathrm{N}=\right.$ and $\left.\mathrm{C}_{5} \mathrm{C}_{5} \mathrm{H}_{4}-\mathrm{N}=\right), 124.7\left(2 \mathrm{C}, \mathrm{C}_{3}\right.$ and $\mathrm{C}_{5}$ pyridine ring), 123.4 (2C, $\mathrm{C}_{2} \mathrm{C}_{5} \mathrm{H}_{4}-\mathrm{N}=$ and $\left.\mathrm{C}_{6} \mathrm{C}_{5} \mathrm{H}_{4}-\mathrm{N}=\right), 123.8$ (1C, $\mathrm{C}_{5}$ pyrazole ring), 119.4 (2C, $\mathrm{C}_{2}$ and $\mathrm{C}_{6}$ aromatic ring), 117.8 (1C, $\mathrm{C}_{4}$ pyrazole ring), $78.3\left(1 \mathrm{C}, \mathrm{C}_{2}\right.$ oxadiazole ring), $21.9\left(1 \mathrm{C}, \mathrm{C}_{6} \mathrm{H}_{4}-\mathrm{CH}_{3}\right), 21.5(1 \mathrm{C}$, $\left.=\mathrm{CH}-\mathrm{CH}_{3}\right)$; LCMS (ESI): m/z $544.2(36.5 \%)\left[\mathrm{M}^{+}\right]$; Anal. calcd. for $\mathrm{C}_{31} \mathrm{H}_{25} \mathrm{~N}_{7} \mathrm{O}_{3}: \mathrm{C}, 68.50 ; \mathrm{H}, 4.64$; N, 18.04; Found: C, 68.55; H, 4.72; N, 18.13\%.

Characterization of 2-chloro- $\mathrm{N}$-(1-(2-(1-phenyl-3-(p-tolyl)-1H-pyrazol-4-yl)-5-(pyridin-4-yl)1,3,4-oxadiazol-3(2H)-yl)ethylidene)aniline: $5 \mathrm{~m}$

IR $\left(\mathrm{KBr}, \mathrm{cm}^{-1}\right):$ 1594, $1539\left(>\mathrm{C}=\mathrm{N}-,>\mathrm{C}=\mathrm{C}<\right.$ stretching), $1353\left(-\mathrm{C}-\mathrm{H}\right.$ stretching, $\left.-\mathrm{C}-\mathrm{CH}_{3}\right)$, $1289\left(>\mathrm{C}=\mathrm{N}\right.$ - stretching, Ar- $\left.\mathrm{NH}_{2}\right), 1220$ (C-O-C stretching, oxadiazole ring), 760 (C-Cl stretching); ${ }^{1} \mathrm{H}$ NMR (DMSO- $\left.\mathrm{d}_{6}, 400 \mathrm{MHz}\right): \delta=8.83\left(\mathrm{~d}, 2 \mathrm{H}, J=8.4 \mathrm{~Hz} \mathrm{C} \mathrm{C}_{2}-\underline{\mathrm{H}}\right.$ and $\mathrm{C}_{6}-\underline{\mathrm{H}}$ pyridine ring), $8.08(\mathrm{~d}$, $2 \mathrm{H}, J=7.4 \mathrm{~Hz} \mathrm{C}_{3}-\underline{\mathrm{H}}$ and $\mathrm{C}_{5}-\underline{\mathrm{H}}$ pyridine ring), 7.94 (s, $1 \mathrm{H}, \mathrm{C}_{5}-\underline{\mathrm{H}}$ pyrazole ring), 7.30-7.70 (m, 13H, $\mathrm{Ar}-\underline{\mathrm{H}}), 5.89\left(\mathrm{~s}, 1 \mathrm{H}, \mathrm{C}_{5}-\underline{\mathrm{H}}\right.$ oxadiazole ring), $2.43\left(\mathrm{~s}, 3 \mathrm{H},-\mathrm{CH}_{3}\right), 2.29\left(\mathrm{~s}, 3 \mathrm{H},-\mathrm{C}_{3}-\mathrm{C}=\mathrm{N}\right) ;{ }^{13} \mathrm{C} \mathrm{NMR}$ $\left(100 \mathrm{MHz}, \mathrm{DMSO}-\mathrm{d}_{6}\right): \delta=157.3\left(1 \mathrm{C}, \mathrm{C}_{5}\right.$ oxadiazole ring), $156.0(1 \mathrm{C},-\mathrm{CH}=\mathrm{N}-), 149.2\left(1 \mathrm{C}, \mathrm{C}_{3}\right.$ pyrazole ring), 149.0 (2C, $\mathrm{C}_{2}$ and $\mathrm{C}_{6}$ pyridine ring), $139.8\left(1 \mathrm{C}, \mathrm{C}_{1} \mathrm{C}_{5} \mathrm{H}_{4}-\mathrm{N}=\right), 139.2\left(1 \mathrm{C}, \mathrm{C}_{1}\right.$ aromatic ring), 138.6 (1C, $\mathrm{C}_{4}$ pyridine ring), $131.4\left(1 \mathrm{C}, \mathrm{C}_{4} \mathrm{C}_{6} \mathrm{H}_{4}-\mathrm{CH}_{3}\right), 130.8\left(1 \mathrm{C}, \mathrm{C}_{1} \mathrm{C}_{6} \mathrm{H}_{4}-\mathrm{CH}_{3}\right)$, $130.1\left(1 \mathrm{C}, \mathrm{C}_{3} \mathrm{C}_{5} \mathrm{H}_{4}-\mathrm{N}=\right), 129.9\left(2 \mathrm{C}, \mathrm{C}_{3}\right.$ and $\mathrm{C}_{5}$ aromatic ring), $129.5\left(2 \mathrm{C}, \mathrm{C}_{3}\right.$ and $\left.\mathrm{C}_{5} \mathrm{C}_{6} \mathrm{H}_{4}-\mathrm{CH}_{3}\right)$, $128.4\left(1 \mathrm{C}, \mathrm{C}_{4} \mathrm{C}_{5} \mathrm{H}_{4}-\mathrm{N}=\right), 128.3\left(1 \mathrm{C}, \mathrm{C}_{5} \mathrm{C}_{5} \mathrm{H}_{4}-\mathrm{N}=\right), 127.5\left(1 \mathrm{C}, \mathrm{C}_{2} \mathrm{C}_{5} \mathrm{H}_{4}-\mathrm{N}=\right), 126.7\left(1 \mathrm{C}, \mathrm{C}_{4}\right.$ aromatic ring), $125.5\left(2 \mathrm{C}, \mathrm{C}_{2}\right.$ and $\left.\mathrm{C}_{6} \mathrm{C}_{6} \mathrm{H}_{4}-\mathrm{CH}_{3}\right), 124.9$ (2C, $\mathrm{C}_{3}$ and $\mathrm{C}_{5}$ pyridine ring), $123.8\left(1 \mathrm{C}, \mathrm{C}_{5}\right.$ pyrazole ring), 119.4 (2C, $\mathrm{C}_{2}$ and $\mathrm{C}_{6}$ aromatic ring), $118.7\left(1 \mathrm{C}, \mathrm{C}_{6} \mathrm{C}_{5} \mathrm{H}_{4}-\mathrm{N}=\right), 117.7$ ( $1 \mathrm{C}, \mathrm{C}_{5}$ pyrazole ring), 
78.6 (1C, $\mathrm{C}_{2}$ oxadiazole ring), $21.8\left(1 \mathrm{C}, \mathrm{C}_{6} \mathrm{H}_{4}-\mathrm{CH}_{3}\right), 21.3\left(1 \mathrm{C},=\mathrm{CH}-\mathrm{CH}_{3}\right) ; \mathrm{LCMS}(\mathrm{ESI}): \mathrm{m} / \mathrm{z} 534.2$ (38.5\%) $\left[\mathrm{M}^{+}\right]$; Anal. calcd. for $\mathrm{C}_{31} \mathrm{H}_{25} \mathrm{ClN}_{6} \mathrm{O}$ : C, 69.85; H, 4.73; N, 15.77; Found: C, 69.89; H, 4.77; $\mathrm{N}, 15.79 \%$.

Characterization of 3-chloro- $N$-(1-(2-(1-phenyl-3-(p-tolyl)-1H-pyrazol-4-yl)-5-(pyridin-4-yl)1,3,4-oxadiazol-3(2H)-yl)ethylidene)aniline: $5 \mathrm{n}$

IR $\left(\mathrm{KBr}, \mathrm{cm}^{-1}\right): 1587,1535\left(>\mathrm{C}=\mathrm{N}-,>\mathrm{C}=\mathrm{C}<\right.$ stretching), 1350 (-C-H stretching, $\left.-\mathrm{C}-\mathrm{CH}_{3}\right)$, $1282\left(>\mathrm{C}=\mathrm{N}\right.$ - stretching, Ar- $\left.\mathrm{NH}_{2}\right), 1225$ (C-O-C stretching, oxadiazole ring), 740 (C-Cl stretching); ${ }^{1} \mathrm{H}$ NMR (DMSO- $\left.\mathrm{d}_{6}, 400 \mathrm{MHz}\right): \delta=8.85\left(\mathrm{~d}, 2 \mathrm{H}, J=8.3 \mathrm{~Hz} \mathrm{C} \mathrm{C}_{2}-\underline{\mathrm{H}}\right.$ and $\mathrm{C}_{6}-\underline{\mathrm{H}}$ pyridine ring), 8.11 (d, $2 \mathrm{H}, J=7.4 \mathrm{~Hz} \mathrm{C}_{3}-\underline{\mathrm{H}}$ and $\mathrm{C}_{5}-\underline{\mathrm{H}}$ pyridine ring), $7.95\left(\mathrm{~s}, 1 \mathrm{H}, \mathrm{C}_{5}-\underline{\mathrm{H}}\right.$ pyrazole ring), 6.90-7.62 (m, $13 \mathrm{H}$, $\mathrm{Ar}-\underline{\mathrm{H}}), 5.93\left(\mathrm{~s}, 1 \mathrm{H}, \mathrm{C}_{5}-\underline{\mathrm{H}}\right.$ oxadiazole ring), $2.44\left(\mathrm{~s}, 3 \mathrm{H},-\mathrm{CH}_{3}\right), 2.30\left(\mathrm{~s}, 3 \mathrm{H},-\mathrm{CH}_{3}-\mathrm{C}=\mathrm{N}\right) ;{ }^{13} \mathrm{C} \mathrm{NMR}$ $\left(100 \mathrm{MHz}, \mathrm{DMSO}-\mathrm{d}_{6}\right): \delta=157.8\left(1 \mathrm{C}, \mathrm{C}_{5}\right.$ oxadiazole ring), $156.1(1 \mathrm{C},-\mathrm{CH}=\mathrm{N}-), 150.4\left(1 \mathrm{C}, \mathrm{C}_{1}\right.$ $\left.\mathrm{C}_{5} \mathrm{H}_{4}-\mathrm{N}=\right), 149.4$ (2C, $\mathrm{C}_{2}$ and $\mathrm{C}_{6}$ pyridine ring), 149.1 (1C, $\mathrm{C}_{3}$ pyrazole ring), $139.3\left(1 \mathrm{C}, \mathrm{C}_{1} \mathrm{C}_{5} \mathrm{H}_{4-}\right.$ $\mathrm{N}=), 138.0\left(1 \mathrm{C}, \mathrm{C}_{4}\right.$ pyridine ring), $134.7\left(1 \mathrm{C}, \mathrm{C}_{3} \mathrm{C}_{5} \mathrm{H}_{4}-\mathrm{N}=\right), 131.9\left(1 \mathrm{C}, \mathrm{C}_{5} \mathrm{C}_{5} \mathrm{H}_{4}-\mathrm{N}=\right), 131.0\left(1 \mathrm{C}, \mathrm{C}_{4}\right.$ $\left.\mathrm{C}_{6} \mathrm{H}_{4}-\mathrm{CH}_{3}\right), 130.4$ (1C, $\left.\mathrm{C}_{1} \mathrm{C}_{6} \mathrm{H}_{4}-\mathrm{CH}_{3}\right), 129.8$ (2C, $\mathrm{C}_{3}$ and $\mathrm{C}_{5}$ aromatic ring), 129.2 (2C, $\mathrm{C}_{3}$ and $\mathrm{C}_{5}$ $\left.\mathrm{C}_{6} \mathrm{H}_{4}-\mathrm{CH}_{3}\right), 127.9\left(1 \mathrm{C}, \mathrm{C}_{4} \mathrm{C}_{5} \mathrm{H}_{4}-\mathrm{N}=\right), 126.7$ (1C, $\mathrm{C}_{4}$ aromatic ring), 125.1 (2C, $\mathrm{C}_{2}$ and $\mathrm{C}_{6} \mathrm{C}_{6} \mathrm{H}_{4}-$ $\left.\mathrm{CH}_{3}\right), 124.0$ (2C, $\mathrm{C}_{3}$ and $\mathrm{C}_{5}$ pyridine ring), 123.7 (1C, $\mathrm{C}_{5}$ pyrazole ring), $122.4\left(1 \mathrm{C}, \mathrm{C}_{2} \mathrm{C}_{6} \mathrm{H}_{4}-\mathrm{CH}_{3}\right)$, 120.7 (1C, $\left.\mathrm{C}_{6} \mathrm{C}_{6} \mathrm{H}_{4}-\mathrm{CH}_{3}\right), 119.5$ (2C, $\mathrm{C}_{2}$ and $\mathrm{C}_{6}$ aromatic ring), 117.1 (1C, $\mathrm{C}_{4}$ pyrazole ring), 78.2 (1C, $\mathrm{C}_{2}$ oxadiazole ring), $21.8\left(1 \mathrm{C}, \mathrm{C}_{6} \mathrm{H}_{4}-\mathrm{CH}_{3}\right), 21.7\left(1 \mathrm{C},=\mathrm{CH}-\mathrm{CH}_{3}\right) ; \mathrm{LCMS}(\mathrm{ESI}): \mathrm{m} / \mathrm{z} 534.2$ (38.5\%) $\left[\mathrm{M}^{+}\right]$; Anal. calcd. for $\mathrm{C}_{31} \mathrm{H}_{25} \mathrm{ClN}_{6} \mathrm{O}$ : C, 69.85; H, 4.73; N, 15.77; Found: C, 69.90; H, 4.77; $\mathrm{N}, 15.82 \%$.

Characterization of 4-chloro- $N$-(1-(2-(1-phenyl-3-(p-tolyl)-1 $H$-pyrazol-4-yl)-5-(pyridin-4-yl)1,3,4-oxadiazol-3(2H)-yl)ethylidene)aniline: 50

IR $\left(\mathrm{KBr}, \mathrm{cm}^{-1}\right): 1582,1537\left(>\mathrm{C}=\mathrm{N}-,>\mathrm{C}=\mathrm{C}<\right.$ stretching), $1356\left(-\mathrm{C}-\mathrm{H}\right.$ stretching, $\left.-\mathrm{C}-\mathrm{CH}_{3}\right)$, $1288\left(>\mathrm{C}=\mathrm{N}\right.$ - stretching, Ar- $\mathrm{NH}_{2}$ ), 1228 (C-O-C stretching, oxadiazole ring), 752 (C-Cl stretching); ${ }^{1} \mathrm{H}$ NMR (DMSO- $\left.\mathrm{d}_{6}, 400 \mathrm{MHz}\right): \delta=8.80\left(\mathrm{~d}, 2 \mathrm{H}, J=8.3 \mathrm{~Hz} \mathrm{C} \mathrm{C}_{2}-\underline{\mathrm{H}}\right.$ and $\mathrm{C}_{6}-\underline{\mathrm{H}}$ pyridine ring), 8.13 (d, $2 \mathrm{H}, J=7.0 \mathrm{~Hz} \mathrm{C}_{3}-\underline{\mathrm{H}}$ and $\mathrm{C}_{5}-\underline{\mathrm{H}}$ pyridine ring), $7.96\left(\mathrm{~s}, 1 \mathrm{H}, \mathrm{C}_{5}-\underline{\mathrm{H}}\right.$ pyrazole ring), 7.10-7.63 (m, 13H, $\mathrm{Ar}-\underline{\mathrm{H}}), 5.91\left(\mathrm{~s}, 1 \mathrm{H}, \mathrm{C}_{5}-\underline{\mathrm{H}}\right.$ oxadiazole ring), $2.44\left(\mathrm{~s}, 3 \mathrm{H},-\mathrm{CH}_{3}\right), 2.31\left(\mathrm{~s}, 3 \mathrm{H},-\mathrm{CH}_{3}-\mathrm{C}=\mathrm{N}\right) ;{ }^{13} \mathrm{C} \mathrm{NMR}$ $\left(100 \mathrm{MHz}, \mathrm{DMSO}-\mathrm{d}_{6}\right): \delta=157.1\left(1 \mathrm{C}, \mathrm{C}_{5}\right.$ oxadiazole ring), $156.4(1 \mathrm{C},-\mathrm{CH}=\mathrm{N}-), 150.2\left(1 \mathrm{C}, \mathrm{C}_{1}\right.$ $\left.\mathrm{C}_{5} \mathrm{H}_{4}-\mathrm{N}=\right), 149.8$ (2C, $\mathrm{C}_{2}$ and $\mathrm{C}_{6}$ pyridine ring), 149.2 (1C, $\mathrm{C}_{3}$ pyrazole ring), 139.1 (1C, $\mathrm{C}_{1}$ aromatic ring), $138.7\left(1 \mathrm{C}, \mathrm{C}_{4}\right.$ pyridine ring), $132.4\left(1 \mathrm{C}, \mathrm{C}_{4} \mathrm{C}_{5} \mathrm{H}_{4}-\mathrm{N}=\right), 131.7\left(1 \mathrm{C}, \mathrm{C}_{4} \mathrm{C}_{6} \mathrm{H}_{4}-\mathrm{CH}_{3}\right)$, $130.8\left(2 \mathrm{C}, \mathrm{C}_{5}\right.$ and $\left.\mathrm{C}_{3} \mathrm{C}_{5} \mathrm{H}_{4}-\mathrm{N}=\right), 130.2\left(1 \mathrm{C}, \mathrm{C}_{1} \mathrm{C}_{6} \mathrm{H}_{4}-\mathrm{CH}_{3}\right), 129.7\left(2 \mathrm{C}, \mathrm{C}_{3}\right.$ and $\left.\mathrm{C}_{5} \mathrm{C}_{6} \mathrm{H}_{4}-\mathrm{CH}_{3}\right), 129.4$ (2C, $\mathrm{C}_{3}$ and $\mathrm{C}_{5}$ aromatic ring), 126.7 (1C, $\mathrm{C}_{4}$ aromatic ring), $125.2\left(2 \mathrm{C}, \mathrm{C}_{2}\right.$ and $\left.\mathrm{C}_{6} \mathrm{C}_{6} \mathrm{H}_{4}-\mathrm{CH}_{3}\right), 124.8$ (2C, $\mathrm{C}_{3}$ and $\mathrm{C}_{5}$ pyridine ring), 123.7 ( $1 \mathrm{C}, \mathrm{C}_{5}$ pyrazole ring), $122.8\left(2 \mathrm{C}, \mathrm{C}_{2}\right.$ and $\left.\mathrm{C}_{6} \mathrm{C}_{6} \mathrm{H}_{4}-\mathrm{CH}_{3}\right), 119.5$ ( $2 \mathrm{C}, \mathrm{C}_{2}$ and $\mathrm{C}_{6}$ aromatic ring), 117.8 ( $1 \mathrm{C}, \mathrm{C}_{5}$ pyrazole ring), 78.0 (1C, $\mathrm{C}_{2}$ oxadiazole ring), 21.9 $\left(1 \mathrm{C}, \mathrm{C}_{6} \mathrm{H}_{4}-\mathrm{CH}_{3}\right), 21.7\left(1 \mathrm{C},=\mathrm{CH}-\mathrm{CH}_{3}\right)$; LCMS (ESI): $\mathrm{m} / \mathrm{z} 534.2(38.5 \%)\left[\mathrm{M}^{+}\right]$; Anal. calcd. for $\mathrm{C}_{31} \mathrm{H}_{25} \mathrm{ClN}_{6} \mathrm{O}: \mathrm{C}, 69.85 ; \mathrm{H}, 4.73$; N, 15.77; Found: C, 69.89; H, 4.78; N, 15.83\%.

Characterization of 2-bromo- $\mathrm{N}$-(1-(2-(1-phenyl-3-(p-tolyl)-1H-pyrazol-4-yl)-5-(pyridin-4-yl)1,3,4-oxadiazol-3(2H)-yl)ethylidene)aniline: $5 p$

IR $\left(\mathrm{KBr}, \mathrm{cm}^{-1}\right): 1588,1531\left(>\mathrm{C}=\mathrm{N}-,>\mathrm{C}=\mathrm{C}<\right.$ stretching), $1356\left(-\mathrm{C}-\mathrm{H}\right.$ stretching, $\left.-\mathrm{C}-\mathrm{CH}_{3}\right)$, $1290\left(>\mathrm{C}=\mathrm{N}\right.$ - stretching, Ar- $\mathrm{NH}_{2}$ ), 1226 (C-O-C stretching, oxadiazole ring), 540 (C-Br stretching); ${ }^{1} \mathrm{H}$ NMR (DMSO- $\left.\mathrm{d}_{6}, 400 \mathrm{MHz}\right): \delta=8.81\left(\mathrm{~d}, 2 \mathrm{H}, J=8.8 \mathrm{~Hz} \mathrm{C}_{2}-\underline{\mathrm{H}}\right.$ and $\mathrm{C}_{6}-\underline{\mathrm{H}}$ pyridine ring), $8.10(\mathrm{~d}$, $2 \mathrm{H}, J=7.8 \mathrm{~Hz} \mathrm{C}_{3}-\underline{\mathrm{H}}$ and $\mathrm{C}_{5}-\underline{\mathrm{H}}$ pyridine ring), 7.98 (s, $1 \mathrm{H}, \mathrm{C}_{5}-\underline{\mathrm{H}}$ pyrazole ring), 7.20-7.75 (m, 13H, $\mathrm{Ar}-\underline{\mathrm{H}}), 5.90\left(\mathrm{~s}, 1 \mathrm{H}, \mathrm{C}_{5}-\underline{\mathrm{H}}\right.$ oxadiazole ring), $2.45\left(\mathrm{~s}, 3 \mathrm{H},-\underline{\mathrm{CH}}_{3}\right), 2.32\left(\mathrm{~s}, 3 \mathrm{H},-\mathrm{C}_{3}-\mathrm{C}=\mathrm{N}\right) ;{ }^{13} \mathrm{C} \mathrm{NMR}$ (100 MHz, DMSO-d $\left.\mathrm{d}_{6}\right): \delta=157.0\left(1 \mathrm{C}, \mathrm{C}_{5}\right.$ oxadiazole ring), $156.9(1 \mathrm{C},-\mathrm{CH}=\mathrm{N}-), 149.5\left(2 \mathrm{C}, \mathrm{C}_{2}\right.$ and $\mathrm{C}_{6}$ pyridine ring), 149.3 (1C, $\mathrm{C}_{3}$ pyrazole ring), $146.7\left(1 \mathrm{C}, \mathrm{C}_{1} \mathrm{C}_{5} \mathrm{H}_{4}-\mathrm{N}=\right), 139.1\left(1 \mathrm{C}, \mathrm{C}_{1}\right.$ aromatic ring), 138.7 (1C, $\mathrm{C}_{4}$ pyridine ring), $132.7\left(1 \mathrm{C}, \mathrm{C}_{3} \mathrm{C}_{5} \mathrm{H}_{4}-\mathrm{N}=\right)$, $131.0\left(1 \mathrm{C}, \mathrm{C}_{4} \mathrm{C}_{6} \mathrm{H}_{4}-\mathrm{CH}_{3}\right), 130.8(1 \mathrm{C}$, $\left.\mathrm{C}_{4} \mathrm{C}_{6} \mathrm{H}_{4}-\mathrm{CH}_{3}\right), 129.8\left(1 \mathrm{C}, \mathrm{C}_{5} \mathrm{C}_{5} \mathrm{H}_{4}-\mathrm{N}=\right), 129.4$ (2C, $\mathrm{C}_{3}$ and $\mathrm{C}_{5}$ aromatic ring), $129.2\left(2 \mathrm{C}, \mathrm{C}_{3}\right.$ and $\mathrm{C}_{5}$ $\mathrm{C}_{6} \mathrm{H}_{4}-\mathrm{CH}_{3}$ ), 126.8 (1C, $\left.\mathrm{C}_{4} \mathrm{C}_{5} \mathrm{H}_{4}-\mathrm{N}=\right), 126.2$ (1C, $\mathrm{C}_{4}$ aromatic ring), 125.0 (2C, $\mathrm{C}_{2}$ and $\mathrm{C}_{6} \mathrm{C}_{6} \mathrm{H}_{4-}$ $\left.\mathrm{CH}_{3}\right), 124.7$ (2C, $\mathrm{C}_{3}$ and $\mathrm{C}_{5}$ pyridine ring), 123.8 (1C, $\mathrm{C}_{5}$ pyrazole ring), $119.2\left(2 \mathrm{C}, \mathrm{C}_{2}\right.$ and $\mathrm{C}_{6}$ aromatic ring), $119.0\left(1 \mathrm{C}, \mathrm{C}_{6} \mathrm{C}_{5} \mathrm{H}_{4}-\mathrm{N}=\right), 118.7\left(1 \mathrm{C}, \mathrm{C}_{2} \mathrm{C}_{5} \mathrm{H}_{4}-\mathrm{N}=\right), 117.8$ (1C, $\mathrm{C}_{4}$ pyrazole ring), 
$78.4\left(1 \mathrm{C}, \mathrm{C}_{2}\right.$ oxadiazole ring), $21.8\left(1 \mathrm{C}, \mathrm{C}_{6} \mathrm{H}_{4}-\mathrm{CH}_{3}\right), 21.7\left(1 \mathrm{C},=\mathrm{CH}-\mathrm{CH}_{3}\right) ; \mathrm{LCMS}$ (ESI): m/z 576.1 (96.3\%) $\left[\mathrm{M}^{+}\right]$; Anal. calcd. for $\mathrm{C}_{31} \mathrm{H}_{25} \mathrm{BrN}_{6} \mathrm{O}: \mathrm{C}, 64.48 ; \mathrm{H}, 4.36 ; \mathrm{N}, 14.55$; Found: $\mathrm{C}, 64.52 ; \mathrm{H}$, $4.41 ; \mathrm{N}, 14.59 \%$.

Characterization of 3-bromo- $N$-(1-(2-(1-phenyl-3-(p-tolyl)-1H-pyrazol-4-yl)-5-(pyridin-4-yl)1,3,4-oxadiazol-3(2H)-yl)ethylidene)aniline: $5 q$

IR $\left(\mathrm{KBr}, \mathrm{cm}^{-1}\right):$ 1597, $1533\left(>\mathrm{C}=\mathrm{N}-,>\mathrm{C}=\mathrm{C}<\right.$ stretching), 1359 (-C-H stretching, $\left.-\mathrm{C}-\mathrm{CH}_{3}\right)$, $1293\left(>\mathrm{C}=\mathrm{N}\right.$ - stretching, Ar-NH$\left.{ }_{2}\right), 1229$ (C-O-C stretching, oxadiazole ring), 565 (C-Br stretching); ${ }^{1} \mathrm{H}$ NMR (DMSO-d $\left.\mathrm{d}_{6}, 400 \mathrm{MHz}\right): \delta=8.79\left(\mathrm{~d}, 2 \mathrm{H}, J=8.8 \mathrm{~Hz} \mathrm{C}_{2}-\underline{\mathrm{H}}\right.$ and $\mathrm{C}_{6}-\underline{\mathrm{H}}$ pyridine ring), 8.14 (d, $2 \mathrm{H}, J=7.8 \mathrm{~Hz} \mathrm{C}_{3}-\underline{\mathrm{H}}$ and $\mathrm{C}_{5}-\underline{\mathrm{H}}$ pyridine ring), 8.03 (s, $1 \mathrm{H}, \mathrm{C}_{5}-\underline{\mathrm{H}}$ pyrazole ring), 7.00-7.72 (m, $13 \mathrm{H}, \mathrm{Ar}-\underline{\mathrm{H}}), 5.89\left(\mathrm{~s}, 1 \overline{\mathrm{H}}, \mathrm{C}_{5}-\underline{\mathrm{H}}\right.$ oxadiazole ring), $2.46\left(\mathrm{~s}, 3 \mathrm{H},-\mathrm{CH}_{3}\right), 2.30\left(\mathrm{~s}, 3 \mathrm{H},-\mathrm{CH}_{3}-\mathrm{C}=\mathrm{N}\right) ;{ }^{13} \mathrm{C}$ NMR (100 MHz, DMSO-d $\left.\mathrm{d}_{6}\right): \delta=157.8\left(1 \mathrm{C}, \mathrm{C}_{5}\right.$ oxadiazole ring), $156.2(1 \mathrm{C},-\mathrm{CH}=\mathrm{N}-), 149.2(2 \mathrm{C}$, $\mathrm{C}_{2}$ and $\mathrm{C}_{6}$ pyridine ring), 149.1 (1C, $\mathrm{C}_{3}$ pyrazole ring), 139.4 (1C, $\mathrm{C}_{1}$ aromatic ring), 138.4 (1C, $\mathrm{C}_{4}$ pyridine ring), $131.4\left(1 \mathrm{C}, \mathrm{C}_{4} \mathrm{C}_{6} \mathrm{H}_{4}-\mathrm{CH}_{3}\right), 130.8\left(1 \mathrm{C}, \mathrm{C}_{5} \mathrm{C}_{5} \mathrm{H}_{4}-\mathrm{N}=\right), 130.1\left(1 \mathrm{C}, \mathrm{C}_{1} \mathrm{C}_{6} \mathrm{H}_{4}-\mathrm{CH}_{3}\right), 129.3$ (2C, $\mathrm{C}_{3}$ and $\mathrm{C}_{5}$ aromatic ring), $129.2\left(2 \mathrm{C}, \mathrm{C}_{3}\right.$ and $\left.\mathrm{C}_{5} \mathrm{C}_{6} \mathrm{H}_{4}-\mathrm{CH}_{3}\right), 126.7$ (1C, $\mathrm{C}_{4}$ aromatic ring), 125.9 $\left(2 \mathrm{C}, \mathrm{C}_{2}\right.$ and $\left.\mathrm{C}_{6} \mathrm{C}_{6} \mathrm{H}_{4}-\mathrm{CH}_{3}\right), 125.1\left(1 \mathrm{C}, \mathrm{C}_{4} \mathrm{C}_{5} \mathrm{H}_{4}-\mathrm{N}=\right), 124.5\left(2 \mathrm{C}, \mathrm{C}_{3}\right.$ and $\mathrm{C}_{5}$ pyridine ring), 123.4 (2C, $\mathrm{C}_{5}$ pyrazole ring and $\left.\mathrm{C}_{3} \mathrm{C}_{5} \mathrm{H}_{4}-\mathrm{N}=\right), 123.2\left(1 \mathrm{C}, \mathrm{C}_{2} \mathrm{C}_{5} \mathrm{H}_{4}-\mathrm{N}=\right), 121.7\left(1 \mathrm{C}, \mathrm{C}_{6} \mathrm{C}_{5} \mathrm{H}_{4}-\mathrm{N}=\right), 119.2$ (2C, $\mathrm{C}_{2}$ and $\mathrm{C}_{6}$ aromatic ring), 117.3 (1C, $\mathrm{C}_{4}$ pyrazole ring), 78.5 (1C, $\mathrm{C}_{2}$ oxadiazole ring), 21.9 $\left(1 \mathrm{C}, \mathrm{C}_{6} \mathrm{H}_{4}-\mathrm{CH}_{3}\right), 21.5\left(1 \mathrm{C},=\mathrm{CH}-\mathrm{CH}_{3}\right)$; LCMS (ESI): m/z $576.1(96.3 \%)\left[\mathrm{M}^{+}\right]$; Anal. calcd. for $\mathrm{C}_{31} \mathrm{H}_{25} \mathrm{BrN}_{6} \mathrm{O}: \mathrm{C}, 64.48 ; \mathrm{H}, 4.36$; N, 14.55; Found: C, 64.54; H, 4.39; N, 14.60\%.

Characterization of 4-bromo- $\mathrm{N}$-(1-(2-(1-phenyl-3-(p-tolyl)-1 H-pyrazol-4-yl)-5-(pyridin-4-yl)1,3,4-oxadiazol-3(2H)-yl)ethylidene)aniline: $5 \mathrm{r}$

IR $\left(\mathrm{KBr}, \mathrm{cm}^{-1}\right):$ 1594, $1536\left(>\mathrm{C}=\mathrm{N}-,>\mathrm{C}=\mathrm{C}<\right.$ stretching), 1353 (-C-H stretching, $\left.-\mathrm{C}-\mathrm{CH}_{3}\right)$, $1290\left(>\mathrm{C}=\mathrm{N}\right.$ - stretching, Ar-NH$\left.H_{2}\right), 1232$ (C-O-C stretching, oxadiazole ring), 550 (C-Br stretching);

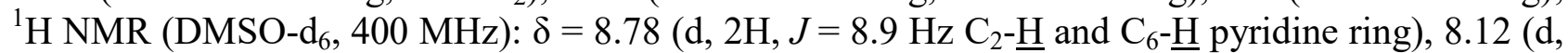
$2 \mathrm{H}, J=7.2 \mathrm{~Hz} \mathrm{C}_{3}-\underline{\mathrm{H}}$ and $\mathrm{C}_{5}-\underline{\mathrm{H}}$ pyridine ring), 8.04 (s, $1 \mathrm{H}, \mathrm{C}_{5}-\underline{\mathrm{H}}$ pyrazole ring), 7.25-7.78 (m, $13 \mathrm{H}$, $\mathrm{Ar}-\underline{\mathrm{H}}), 5.87\left(\mathrm{~s}, 1 \mathrm{H}, \mathrm{C}_{5}-\underline{\mathrm{H}}\right.$ oxadiazole ring), $2.44\left(\mathrm{~s}, 3 \mathrm{H},-\mathrm{CH}_{3}\right), 2.32\left(\mathrm{~s}, 3 \mathrm{H},-\mathrm{CH}_{3}-\mathrm{C}=\mathrm{N}\right) ;{ }^{13} \mathrm{C} \mathrm{NMR}$ $\left(100 \mathrm{MHz}, \mathrm{DMSO}-\mathrm{d}_{6}\right): \delta=157.6\left(1 \mathrm{C}, \mathrm{C}_{5}\right.$ oxadiazole ring), $156.1(1 \mathrm{C},-\mathrm{CH}=\mathrm{N}-), 151.5\left(1 \mathrm{C}, \mathrm{C}_{1}\right.$ $\mathrm{C}_{5} \mathrm{H}_{4}-\mathrm{N}=$ ), 149.8 (2C, $\mathrm{C}_{2}$ and $\mathrm{C}_{6}$ pyridine ring), 149.4 (1C, $\mathrm{C}_{3}$ pyrazole ring), 139.3 (1C, $\mathrm{C}_{1}$ aromatic ring), 138.7 (1C, $\mathrm{C}_{4}$ pyridine ring), $132.0\left(2 \mathrm{C}, \mathrm{C}_{3}\right.$ and $\left.\mathrm{C}_{5} \mathrm{C}_{5} \mathrm{H}_{4}-\mathrm{N}=\right), 131.0\left(1 \mathrm{C}, \mathrm{C}_{4} \mathrm{C}_{6} \mathrm{H}_{4}-\right.$ $\left.\mathrm{CH}_{3}\right), 130.7\left(1 \mathrm{C}, \mathrm{C}_{1} \mathrm{C}_{6} \mathrm{H}_{4}-\mathrm{CH}_{3}\right), 129.4\left(2 \mathrm{C}, \mathrm{C}_{3}\right.$ and $\left.\mathrm{C}_{5} \mathrm{C}_{6} \mathrm{H}_{4}-\mathrm{CH}_{3}\right), 129.2\left(2 \mathrm{C}, \mathrm{C}_{3}\right.$ and $\mathrm{C}_{5}$ aromatic ring), 126.1 (1C, $\mathrm{C}_{4}$ aromatic ring), $125.3\left(2 \mathrm{C}, \mathrm{C}_{3}\right.$ and $\left.\mathrm{C}_{5} \mathrm{C}_{6} \mathrm{H}_{4}-\mathrm{CH}_{3}\right), 124.7\left(2 \mathrm{C}, \mathrm{C}_{3}\right.$ and $\mathrm{C}_{5}$ pyridine ring), 123.7 ( $1 \mathrm{C}, \mathrm{C}_{5}$ pyrazole ring), $122.7\left(2 \mathrm{C}, \mathrm{C}_{2}\right.$ and $\left.\mathrm{C}_{6} \mathrm{C}_{5} \mathrm{H}_{4}-\mathrm{N}=\right), 121.4\left(1 \mathrm{C}, \mathrm{C}_{4} \mathrm{C}_{5} \mathrm{H}_{4}-\mathrm{N}=\right), 119.2$ (2C, $\mathrm{C}_{2}$ and $\mathrm{C}_{6}$ aromatic ring), 117.1 ( $1 \mathrm{C}, \mathrm{C}_{4}$ pyrazole ring), 78.7 (1C, $\mathrm{C}_{2}$ oxadiazole ring), 21.7 $\left(1 \mathrm{C}, \mathrm{C}_{6} \mathrm{H}_{4}-\mathrm{CH}_{3}\right), 21.1\left(1 \mathrm{C},=\mathrm{CH}-\mathrm{CH}_{3}\right)$; LCMS (ESI): m/z $576.1(96.3 \%)\left[\mathrm{M}^{+}\right]$; Anal. calcd. for $\mathrm{C}_{31} \mathrm{H}_{25} \mathrm{BrN}_{6} \mathrm{O}: \mathrm{C}, 64.48 ; \mathrm{H}, 4.36$; N, 14.55; Found: C, 64.50; H, 4.40; N, 14.61\%.

\section{Characterization of 4-bromo-2-methyl- $N$-(1-(2-(1-phenyl-3-(p-tolyl)-1 H-pyrazol-4-yl)-5- (pyridin-4-yl)-1,3,4-oxadiazol-3(2H)-yl)ethylidene)aniline: $5 \mathrm{~s}$}

IR $\left(\mathrm{KBr}, \mathrm{cm}^{-1}\right): 1599,1545\left(>\mathrm{C}=\mathrm{N}-,>\mathrm{C}=\mathrm{C}<\right.$ stretching), $1356\left(-\mathrm{C}-\mathrm{H}\right.$ stretching, $\left.-\mathrm{C}-\mathrm{CH}_{3}\right)$, $1294\left(>\mathrm{C}=\mathrm{N}\right.$ - stretching, Ar- $\left.\mathrm{NH}_{2}\right), 1238$ (C-O-C stretching, oxadiazole ring), 555 (C-Br stretching); ${ }^{1} \mathrm{H}$ NMR (DMSO- $\left.\mathrm{d}_{6}, 400 \mathrm{MHz}\right): \delta=8.79\left(\mathrm{~d}, 2 \mathrm{H}, J=8.2 \mathrm{~Hz} \mathrm{C}_{2}-\underline{\mathrm{H}}\right.$ and $\mathrm{C}_{6}-\underline{\mathrm{H}}$ pyridine ring), $8.15(\mathrm{~d}$, $2 \mathrm{H}, J=7.4 \mathrm{~Hz} \mathrm{C}_{3}-\underline{\mathrm{H}}$ and $\mathrm{C}_{5}-\underline{\mathrm{H}}$ pyridine ring), 8.07 (s, $1 \mathrm{H}, \mathrm{C}_{5}-\underline{\mathrm{H}}$ pyrazole ring), 7.15-7.72 (m, $12 \mathrm{H}$, $\mathrm{Ar}-\underline{\mathrm{H}}), 5.87\left(\mathrm{~s}, 1 \mathrm{H}, \mathrm{C}_{5}-\underline{\mathrm{H}}\right.$ oxadiazole ring), $2.42\left(\mathrm{~s}, 3 \mathrm{H},-\mathrm{CH}_{3}\right), 2.33\left(\mathrm{~s}, 3 \mathrm{H},-\mathrm{CH}_{3}-\mathrm{C}=\mathrm{N}\right) ;{ }^{13} \mathrm{C} \mathrm{NMR}$ $\left(100 \mathrm{MHz}, \mathrm{DMSO}-\mathrm{d}_{6}\right): \delta=157.2\left(1 \mathrm{C}, \mathrm{C}_{5}\right.$ oxadiazole ring), $156.7(1 \mathrm{C},-\mathrm{CH}=\mathrm{N}-), 149.8\left(1 \mathrm{C}, \mathrm{C}_{3}\right.$ pyrazole ring), $149.5\left(2 \mathrm{C}, \mathrm{C}_{2}\right.$ and $\mathrm{C}_{6}$ pyridine ring), $139.6\left(1 \mathrm{C}, \mathrm{C}_{1}\right.$ aromatic ring), $138.3\left(1 \mathrm{C}, \mathrm{C}_{4}\right.$ pyridine ring), $135.7\left(1 \mathrm{C}, \mathrm{C}_{3} \mathrm{C}_{5} \mathrm{H}_{3}-\mathrm{N}=\right), 132.0\left(1 \mathrm{C}, \mathrm{C}_{2} \mathrm{C}_{5} \mathrm{H}_{3}-\mathrm{N}=\right), 131.9\left(1 \mathrm{C}, \mathrm{C}_{4} \mathrm{C}_{6} \mathrm{H}_{4}-\mathrm{CH}_{3}\right), 130.0$ $\left(1 \mathrm{C}, \mathrm{C}_{1} \mathrm{C}_{6} \mathrm{H}_{4}-\mathrm{CH}_{3}\right), 129.8\left(1 \mathrm{C}, \mathrm{C}_{5} \mathrm{C}_{5} \mathrm{H}_{3}-\mathrm{N}=\right), 129.4\left(2 \mathrm{C}, \mathrm{C}_{3}\right.$ and $\left.\mathrm{C}_{5} \mathrm{C}_{6} \mathrm{H}_{4}-\mathrm{CH}_{3}\right), 129.1\left(2 \mathrm{C}, \mathrm{C}_{3}\right.$ and $\mathrm{C}_{5}$ aromatic ring), $127.8\left(1 \mathrm{C}, \mathrm{C}_{1} \mathrm{C}_{5} \mathrm{H}_{3}-\mathrm{N}=\right), 126.4$ (1C, $\mathrm{C}_{4}$ aromatic ring), $125.2\left(2 \mathrm{C}, \mathrm{C}_{2}\right.$ and $\mathrm{C}_{6}$ $\left.\mathrm{C}_{6} \mathrm{H}_{4}-\mathrm{CH}_{3}\right), 124.8\left(2 \mathrm{C}, \mathrm{C}_{3}\right.$ and $\mathrm{C}_{5}$ pyridine ring), $124.2\left(1 \mathrm{C}, \mathrm{C}_{6} \mathrm{C}_{5} \mathrm{H}_{3}-\mathrm{N}=\right), 123.8\left(1 \mathrm{C}, \mathrm{C}_{5}\right.$ pyrazole ring), $121.1\left(1 \mathrm{C}, \mathrm{C}_{4} \mathrm{C}_{5} \mathrm{H}_{3}-\mathrm{N}=\right), 119.0$ (2C, $\mathrm{C}_{2}$ and $\mathrm{C}_{6}$ aromatic ring), 117.5 (1C, $\mathrm{C}_{4}$ pyrazole ring), $78.2\left(1 \mathrm{C}, \mathrm{C}_{2}\right.$ oxadiazole ring), $21.5\left(1 \mathrm{C}, \mathrm{C}_{6} \mathrm{H}_{4}-\mathrm{CH}_{3}\right), 21.4\left(1 \mathrm{C},=\mathrm{CH}-\mathrm{CH}_{3}\right), 17.3\left(1 \mathrm{C},-\mathrm{CH}_{3}\right) ; \mathrm{LCMS}$ 
(ESI): $\mathrm{m} / \mathrm{z} 591.1$ (35.7\%) $\left[\mathrm{M}^{+}\right]$; Anal. calcd. for $\mathrm{C}_{32} \mathrm{H}_{27} \mathrm{BrN}_{6} \mathrm{O}: \mathrm{C}, 64.98 ; \mathrm{H}, 4.60 ; \mathrm{N}, 14.21$; Found: C, 65.05; H, 4.63; N, 14.26\%.

\section{3-chloro-4-fluoro- $N$-(1-(2-(1-phenyl-3-(p-tolyl)-1H-pyrazol-4-yl)-5-(pyridin-4-yl)-1,3,4- oxadiazol-3(2H)-yl)ethylidene)aniline: $5 \mathrm{t}$}

IR $\left(\mathrm{KBr}, \mathrm{cm}^{-1}\right): 1595,1544\left(>\mathrm{C}=\mathrm{N}-,>\mathrm{C}=\mathrm{C}<\right.$ stretching), 1359 (-C-H stretching, $\left.-\mathrm{C}-\mathrm{CH}_{3}\right)$, 1291 ( $>\mathrm{C}=\mathrm{N}$ - stretching, Ar- $\mathrm{NH}_{2}$ ), 1239 (C-O-C stretching, oxadiazole ring), 1150 (C-F stretching), 852 (C-Cl stretching); ${ }^{1} \mathrm{H}$ NMR (DMSO-d $\left.6,400 \mathrm{MHz}\right): \delta=8.81\left(\mathrm{~d}, 2 \mathrm{H}, J=8.9 \mathrm{~Hz} \mathrm{C}{ }_{2}-\underline{\mathrm{H}}\right.$ and $\mathrm{C}_{6}-\underline{\mathrm{H}}$

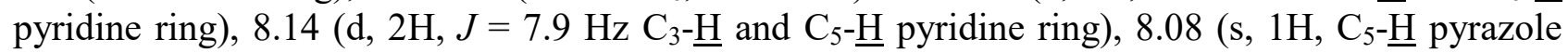
ring), 7.18-7.72 (m, 12H, Ar- $\underline{\mathrm{H}}), 5.86\left(\mathrm{~s}, 1 \mathrm{H}, \mathrm{C}_{5}-\underline{\mathrm{H}}\right.$ oxadiazole ring), $2.40\left(\mathrm{~s}, 3 \mathrm{H},-\underline{\mathrm{C}}_{3}\right), 2.31(\mathrm{~s}, 3 \mathrm{H}$, $\left.-\mathrm{CH}_{3}-\mathrm{C}=\mathrm{N}\right) ;{ }^{13} \mathrm{C}$ NMR $\left(100 \mathrm{MHz}, \mathrm{DMSO}-\mathrm{d}_{6}\right): \delta=157.6\left(1 \mathrm{C}, \mathrm{C}_{5}\right.$ oxadiazole ring), $157.2(1 \mathrm{C}, \mathrm{JC}-\mathrm{F}$ $\left.233.4 \mathrm{c} / \mathrm{sec}, \mathrm{C}_{4} \mathrm{C}_{5} \mathrm{H}_{3}-\mathrm{N}=\right), 156.3(1 \mathrm{C},-\mathrm{CH}=\mathrm{N}-), 149.7$ (1C, $\mathrm{C}_{3}$ pyrazole ring), $149.4\left(2 \mathrm{C}_{2} \mathrm{C}_{2}\right.$ and $\mathrm{C}_{6}$ pyridine ring), $146.4\left(1 \mathrm{C}, \mathrm{C}_{1} \mathrm{C}_{5} \mathrm{H}_{3}-\mathrm{N}=\right), 139.5$ (1C, $\mathrm{C}_{1}$ aromatic ring), 138.4 (1C, $\mathrm{C}_{4}$ pyridine ring), $133.4,131.2\left(1 \mathrm{C}, \mathrm{C}_{4} \mathrm{C}_{6} \mathrm{H}_{4}-\mathrm{CH}_{3}\right), 130.9\left(1 \mathrm{C}, \mathrm{C}_{1} \mathrm{C}_{6} \mathrm{H}_{4}-\mathrm{CH}_{3}\right), 129.4\left(2 \mathrm{C}, \mathrm{C}_{3}\right.$ and $\left.\mathrm{C}_{5} \mathrm{C}_{6} \mathrm{H}_{4}-\mathrm{CH}_{3}\right)$, 129.2 (2C, $\mathrm{C}_{3}$ and $\mathrm{C}_{5}$ aromatic ring), 126.7 (1C, $\mathrm{C}_{4}$ aromatic ring), $125.9\left(2 \mathrm{C}, \mathrm{C}_{2}\right.$ and $\mathrm{C}_{6} \mathrm{C}_{6} \mathrm{H}_{4}-\mathrm{CH}_{3}$ ), 124.9 (2C, $\mathrm{C}_{3}$ and $\mathrm{C}_{5}$ pyridine ring), 124.5 (1C, $\left.\mathrm{C}_{2} \mathrm{C}_{5} \mathrm{H}_{3}-\mathrm{N}=\right), 123.7$ (1C, $\mathrm{C}_{5}$ pyrazole ring), 122.8 $\left(2 \mathrm{C}, \mathrm{C}_{3}\right.$ and $\left.\mathrm{C}_{6} \mathrm{C}_{5} \mathrm{H}_{3}-\mathrm{N}=\right), 119.0\left(2 \mathrm{C}, \mathrm{C}_{2}\right.$ and $\mathrm{C}_{6}$ aromatic ring), $118.9\left(1 \mathrm{C}, \mathrm{C}_{5} \mathrm{C}_{5} \mathrm{H}_{3}-\mathrm{N}=\right), 117.5(1 \mathrm{C}$, $\mathrm{C}_{4}$ pyrazole ring), $78.5\left(1 \mathrm{C}, \mathrm{C}_{2}\right.$ oxadiazole ring), $21.9\left(1 \mathrm{C}, \mathrm{C}_{6} \mathrm{H}_{4}-\mathrm{CH}_{3}\right), 21.7\left(1 \mathrm{C},=\mathrm{CH}-\mathrm{CH}_{3}\right)$; LCMS (ESI): $\mathrm{m} / \mathrm{z} 552.2(38.5 \%)\left[\mathrm{M}^{+}\right]$; Anal. calcd. for $\mathrm{C}_{31} \mathrm{H}_{24} \mathrm{ClFN}_{6} \mathrm{O}: \mathrm{C}, 67.57 ; \mathrm{H}, 4.39 ; \mathrm{N}, 15.25$; Found: C, 67.63; H, 4.42; N, 15.32\%.

\section{Antimicrobial assay}

Antimicrobial studies of newly synthesized derivatives 5a-t were carried out against the representative group of Gram positive $S$. aureus (MTCC-96), S. pyogenes (MTCC-442) and Gramnegative $E$. coli (MTCC-443), $P$. aeruginosa (MTCC-1688)) bacterial and fungal strains such as, $C$. albicans (MTCC-227), A. niger (MTCC-282) and A. clavatus (MTCC-1323). The results depicted in Table 2 exposed that most of the tested compounds displayed variable inhibitory effects. These all titled compounds (5a-t) were shown screening at different concentration of 1000, 500, 200, 100, 50 , and $25 \mu \mathrm{g} / \mathrm{mL}$ by broth dilution method [27-29]. In this method 2\% DMSO used as a diluents and standard drugs to test upon standard microbial strains. At $1000 \mu \mathrm{g} / \mathrm{ML}$ concentration synthesized compounds were diluted. The control tube containing no antibiotic was immediately subcultured [before inoculation] by spreading a loopful evenly over quarter of a plate of medium suitable for the growth of test organisms. At $37^{\circ} \mathrm{C}$, for $24 \mathrm{~h}$ the culture tubes were then incubated and the development of bacteria was monitored visually and spectrophotometrically. $10 \mu \mathrm{g} / \mathrm{mL}$ suspensions were more inoculated on a suitable media, after 24 and $48 \mathrm{~h}$ development of bacterial strains was noted. The lowest concentration (the highest dilution) required to arrest the growth of bacteria was regarded as MIC. DMSO and sterilized distilled water were used as negative control, while chloramphenicol antibiotic (1 U strength) was used as positive control. The recently synthesized derivatives 5a-t was displayed for their antifungal activity against in six sets against $C$. albicans (MTCC-227), A. niger (MTCC-282) and A. clavatus (MTCC-1323) at various primary concentrations of 1000,500 and $250 \mu \mathrm{g} \mathrm{ml}^{-1}$. The primary screen active compounds were similarly diluted to obtain $200,125,100,62.5,50,25$ and $12.5 \mu \mathrm{g} \mathrm{ml}^{-1}$ concentrations for secondary screening to test in a second set of dilution against all microorganisms, For antifungal activity griseofulvin used as a standard drug, which showed 500, 100 and $100 \mu \mathrm{g} \mathrm{ml}^{-1} \mathrm{MIC}$ against responsible fungal strains such as $C$. albicans, A. niger and A. clavatus. For development of fungi, in the present method, we have used Sabourauds dextrose broth at $28^{\circ} \mathrm{C}$ in aerobic condition for 48 h. Sterilized distilled water and DMSO and used as negative controls while 'griseofulvin' (1 U strength) was used as a positive control".

\section{Antimicrobial studies}

For antibacterial activity ampicillin used as a standard drug and results displayed in Table 2 . Compounds 5c and $5 \mathrm{l}$ having $\left(\mathrm{C}_{6} \mathrm{H}_{4}-4-\mathrm{F}\right),\left(\mathrm{C}_{6} \mathrm{H}_{4}-4-\mathrm{NO}_{2}\right)$ displayed excellent activity against $E$. coli. In case of gram negative bacteria $P$. aeruginosa, compounds $\mathbf{5 c}\left(\mathrm{C}_{6} \mathrm{H}_{4}-4-\mathrm{F}\right)$ and $\mathbf{5 r}\left(\mathrm{C}_{6} \mathrm{H}_{4}-4-\mathrm{NO}_{2}\right)$ 
exhibited very good and good activity at $\mathrm{MIC}=25$ and $50 \mu \mathrm{g} \mathrm{ml}^{-1}$. Compound $\mathbf{5 t}\left(\mathrm{C}_{6} \mathrm{H}_{3}-3-\mathrm{Cl}-4-\mathrm{F}\right)$ had shown $\mathrm{MIC}=12.5 \mu \mathrm{g} \mathrm{ml}^{-1}$ which observed as the highest inhibition against $S$. pyogenes. Other compounds showed moderate activity against bacterial strains as shown in Table 2. For antifungal activity MIC was carried out on $C$. albicans, $A$. niger and $A$. clavatus by conventional broth dilution method. Griseofulvin was used as a standard drug for antifungal activity at different concentration of $1000,500,250,100,50,25$ and $12.5 \mu \mathrm{g} / \mathrm{mL}$. Excellent and good activity posseded against $C$. albicans and $A$. clavatus by derivatives $5 \mathbf{i}\left(\mathrm{C}_{6} \mathrm{H}_{4}-4-\mathrm{OCH}_{3}\right)$ and $\mathbf{5 f}\left(\mathrm{C}_{6} \mathrm{H}_{4}-4-\mathrm{CH}_{3}\right)$. Other derivatives showed moderate antifungal activity against responsible fungal strains. The results of the antifungal studies are reported in Table 2. Data given in table shown that the presence of functional group at para position amended antibacterial activity as compared to ortho and meta substituted derivatives.

Table 2. Results of antimicrobial activity of compounds (5a-t).

\begin{tabular}{|c|c|c|c|c|c|c|c|c|}
\hline \multirow[t]{2}{*}{$\begin{array}{l}\text { Sr. } \\
\text { no. }\end{array}$} & \multirow[t]{2}{*}{$-\mathbf{A r}$} & \multicolumn{4}{|c|}{$\begin{array}{l}\text { Minimum inhibitory concentration } \\
\text { (MIC) for bacteria }(\mu \mathrm{g} / \mathrm{mL})\end{array}$} & \multicolumn{3}{|c|}{$\begin{array}{c}\text { Minimum inhibitory } \\
\text { concentration } \\
\text { (MIC) for fungi }(\mu \mathrm{g} / \mathrm{mL})\end{array}$} \\
\hline & & E.c. & P.a. & S.a. & S.p. & C.a. & A.n. & A.c. \\
\hline $\mathbf{5 a}$ & $\mathrm{C}_{6} \mathrm{H}_{4}-\mathrm{H}$ & 100 & 125 & 250 & 100 & 1000 & $>1000$ & $>1000$ \\
\hline $5 \mathbf{b}$ & $\mathrm{C}_{6} \mathrm{H}_{4}-2-\mathrm{F}$ & 100 & 1000 & 1000 & 250 & 1000 & 1000 & 1000 \\
\hline $5 c$ & $\mathrm{C}_{6} \mathrm{H}_{4}-4-\mathrm{F}$ & 12.5 & 25 & 1000 & 250 & 500 & $>1000$ & $>1000$ \\
\hline $5 d$ & $\mathrm{C}_{6} \mathrm{H}_{4}-2-\mathrm{CH}_{3}$ & 100 & 125 & 200 & 100 & 250 & 500 & 100 \\
\hline $5 e$ & $\mathrm{C}_{6} \mathrm{H}_{4}-3-\mathrm{CH}_{3}$ & 500 & 250 & 1000 & 500 & 500 & $>1000$ & $>1000$ \\
\hline $5 f$ & $\mathrm{C}_{6} \mathrm{H}_{4}-4-\mathrm{CH}_{3}$ & 200 & 250 & 200 & 25 & 50 & 100 & 50 \\
\hline $5 \mathrm{~g}$ & $\mathrm{C}_{6} \mathrm{H}_{4}-2-\mathrm{OCH}_{3}$ & 100 & 200 & 250 & 250 & $>1000$ & $>1000$ & $>1000$ \\
\hline $5 h$ & $\mathrm{C}_{6} \mathrm{H}_{4}-3-\mathrm{OCH}_{3}$ & 250 & 250 & 1000 & 200 & 500 & 100 & 100 \\
\hline $5 \mathbf{i}$ & $\mathrm{C}_{6} \mathrm{H}_{4}-4-\mathrm{OCH}_{3}$ & 200 & 200 & 250 & 125 & 50 & 1000 & 50 \\
\hline $\mathbf{5 j}$ & $\mathrm{C}_{6} \mathrm{H}_{4}-2-\mathrm{NO}_{2}$ & 250 & 250 & 500 & 200 & $>1000$ & 250 & 1000 \\
\hline $5 \mathbf{k}$ & $\mathrm{C}_{6} \mathrm{H}_{4}-3-\mathrm{NO}_{2}$ & 125 & 125 & 250 & 250 & 250 & 500 & 500 \\
\hline 51 & $\mathrm{C}_{6} \mathrm{H}_{4}-4-\mathrm{NO}_{2}$ & 12.5 & 200 & 250 & 200 & 1000 & 100 & 1000 \\
\hline $5 \mathrm{~m}$ & $\mathrm{C}_{6} \mathrm{H}_{4}-2-\mathrm{Cl}$ & 200 & 125 & 250 & 125 & 1000 & 1000 & 1000 \\
\hline $5 n$ & $\mathrm{C}_{6} \mathrm{H}_{4}-3-\mathrm{Cl}$ & 125 & 125 & 250 & 100 & 500 & 1000 & $>1000$ \\
\hline 50 & $\mathrm{C}_{6} \mathrm{H}_{4}-4-\mathrm{Cl}$ & 100 & 200 & 500 & 100 & 1000 & 25 & 12.5 \\
\hline $5 p$ & $\mathrm{C}_{6} \mathrm{H}_{4}-2-\mathrm{Br}$ & 200 & 250 & 250 & 250 & 1000 & 1000 & 1000 \\
\hline $5 q$ & $\mathrm{C}_{6} \mathrm{H}_{4}-3-\mathrm{Br}$ & 250 & 250 & 500 & 100 & 500 & $>1000$ & $>1000$ \\
\hline $5 r$ & $\mathrm{C}_{6} \mathrm{H}_{4}-4-\mathrm{Br}$ & 100 & 50 & 250 & 250 & $>1000$ & $>1000$ & $>1000$ \\
\hline $5 s$ & $\begin{array}{c}\mathrm{C}_{6} \mathrm{H}_{3}-4-\mathrm{Br} \\
-2-\mathrm{CH}_{3}\end{array}$ & 125 & 200 & 500 & 200 & $>1000$ & $>1000$ & $>1000$ \\
\hline $5 t$ & $\begin{array}{c}\mathrm{C}_{6} \mathrm{H}_{3}-3-\mathrm{Cl} \\
-4-\mathrm{F}\end{array}$ & 100 & 500 & 500 & 12.5 & 250 & $>1000$ & $>1000$ \\
\hline \multicolumn{2}{|c|}{ Ampicillin } & 100 & 100 & 250 & 100 & - & - & - \\
\hline \multicolumn{2}{|c|}{ Griseofulvin } & - & - & - & - & 500 & 100 & 100 \\
\hline
\end{tabular}

E. $\boldsymbol{c}$.- Escherichia coli MTCC 443; P. a.- Pseudomonas aeruginosa MTCC 1688, S. a.Staphylococcus aureus MTCC 96; $\boldsymbol{S}$. p.- Staphylococcus pyogenes MTCC 442; C. a.- Candida albicans MTCC 227; A. n.- Aspergillus niger MTCC 282; A. c.- Aspergillus clavatus MTCC 1323

\section{SAR studies}

SAR studies exposed that the antimicrobial activity in heterocyclic class of pyrazole, 1,3,4oxadiazole and pyridine scaffolds rely on the character of the peripheral substituent and their spatial relationship within this framework. Different electronic environment of the molecules was confirmed by the pattern of substitution. The electronic nature of the substituent escort to important variance in antimicrobial activity. The occurrence of chloro, fluoro and nitro substituents at para situation on aromatic ring arrangement has enlarged the antibacterial activity of compounds 
compared to those of electron withdrawing groups. The presence of electron donating such as methyl and methoxy group on phenyl ring provides a positive effect on antifungal activity.

\section{Conclusion}

We have synthesized novel series of (5a-t) by conventional method with growth in product of reactions. The excellent properties of this new series of titled compounds of antimicrobial activity deserve further examination. The mode of action at molecular level explains the activity observed. The effectiveness and deeper insight in to titled compounds' SAR study committed to show additional physiochemical and biological parameters.

\section{Acknowledgments}

The authors are thankful to the University Grants Commission (UGC) New Delhi, and Department of Science and Technology (DST), New Delhi, for financial support under the NONSAP and DST-FIST programs respectively. N. C. Desai is highly thankful to the UGC, New Delhi for providing a UGC-BSR one-time grant (2012).

\section{Supporting Information}

IR, ${ }^{1} \mathrm{H}$ and ${ }^{13} \mathrm{C}$ NMR and Mass spectra have been provided in supporting information.

\section{Conflict of interest}

There is no conflict of interest.

\section{Supporting Information}

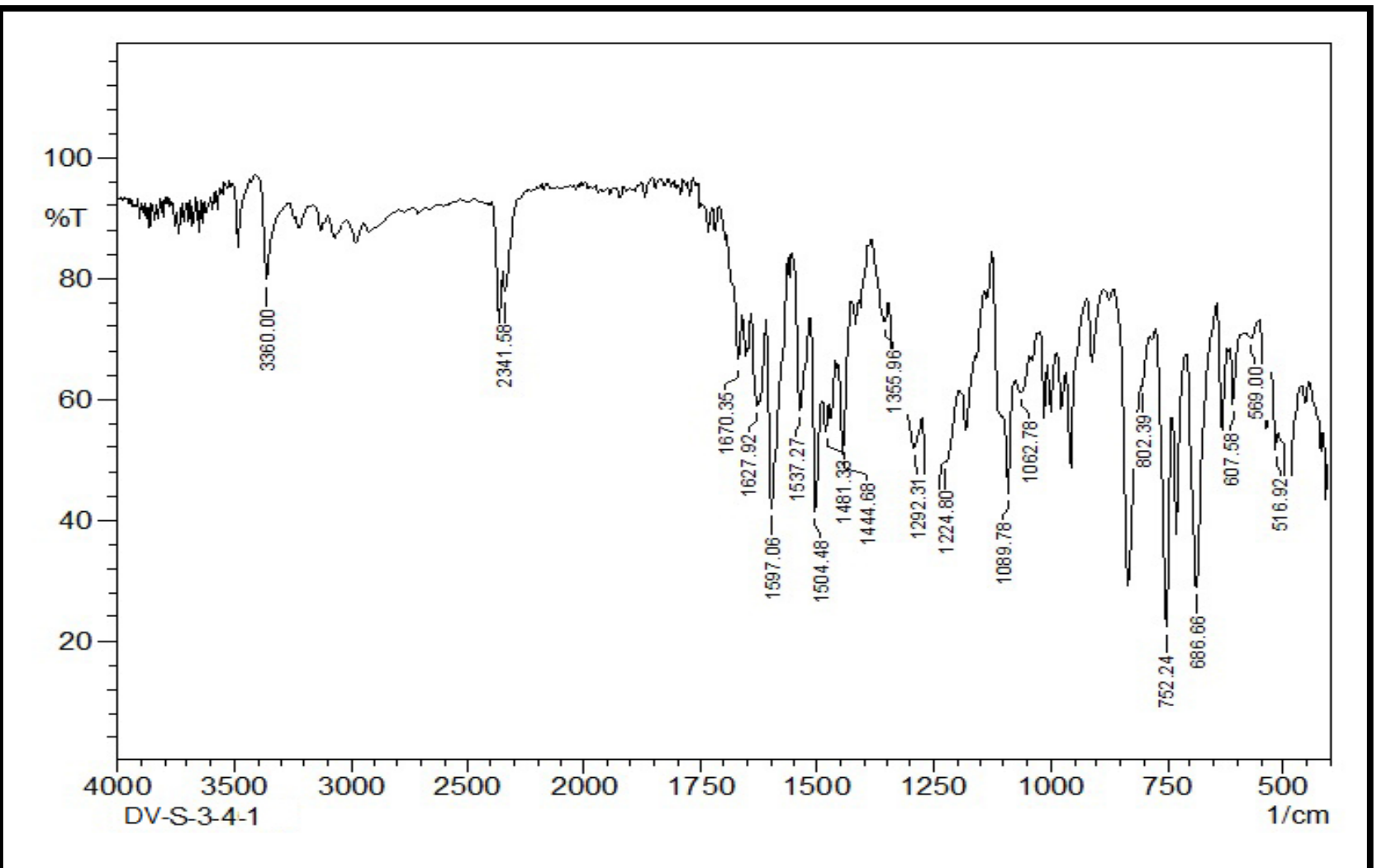

1. IR of -4- $\mathrm{NO}_{2}$ Compound $5 \mathrm{I}$ 
DV3-4

$1 \mathrm{H}$ NMR in DMSO-d6, 500MHz

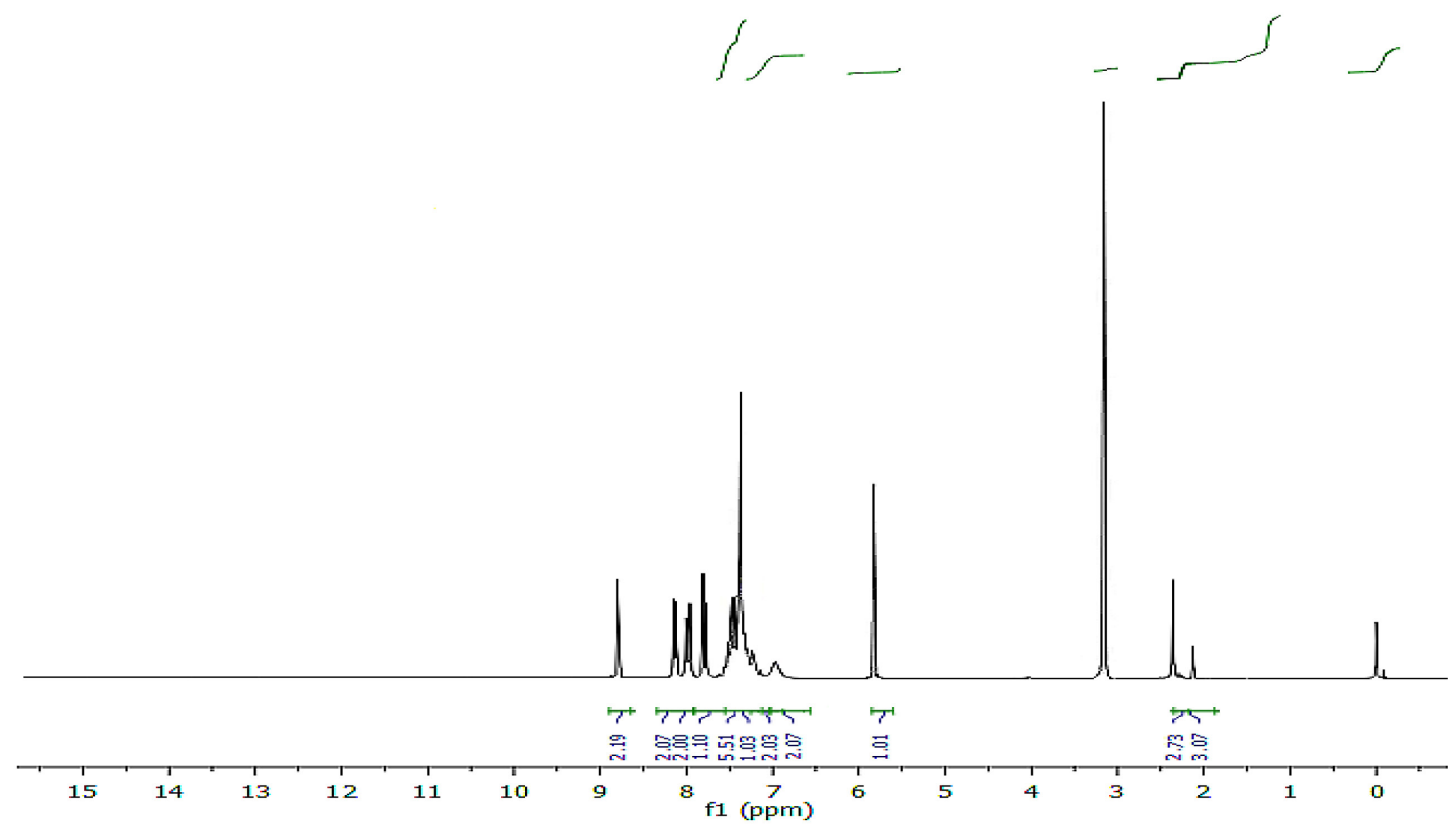

2. ${ }^{1} \mathrm{H}$ NMR of -4-NO ${ }_{2}$ Compound $5 \mathrm{I}$

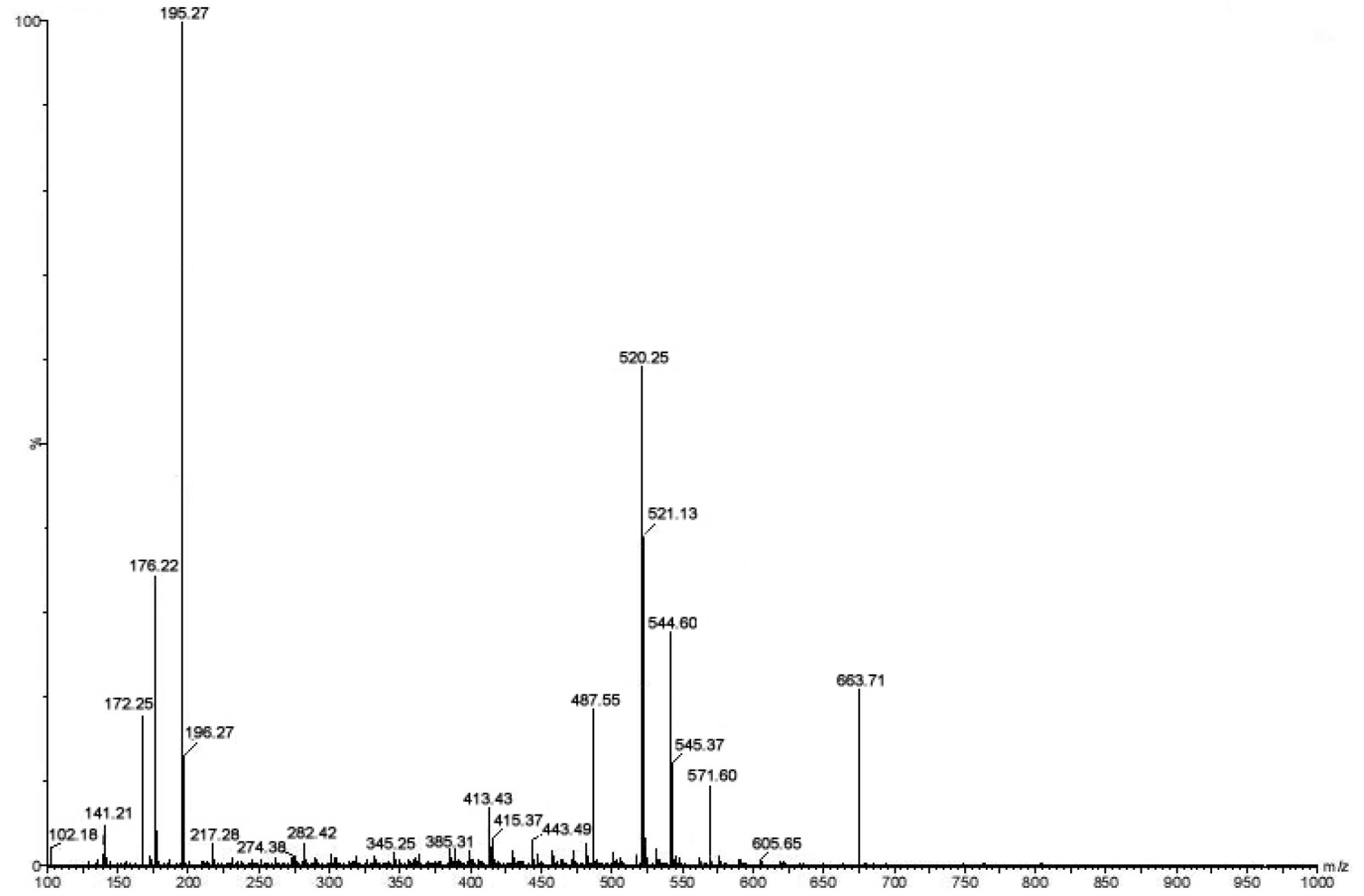

3. Mass of 4-NO $\mathrm{O}_{2}$ Compound $5 \mathrm{I}$ 


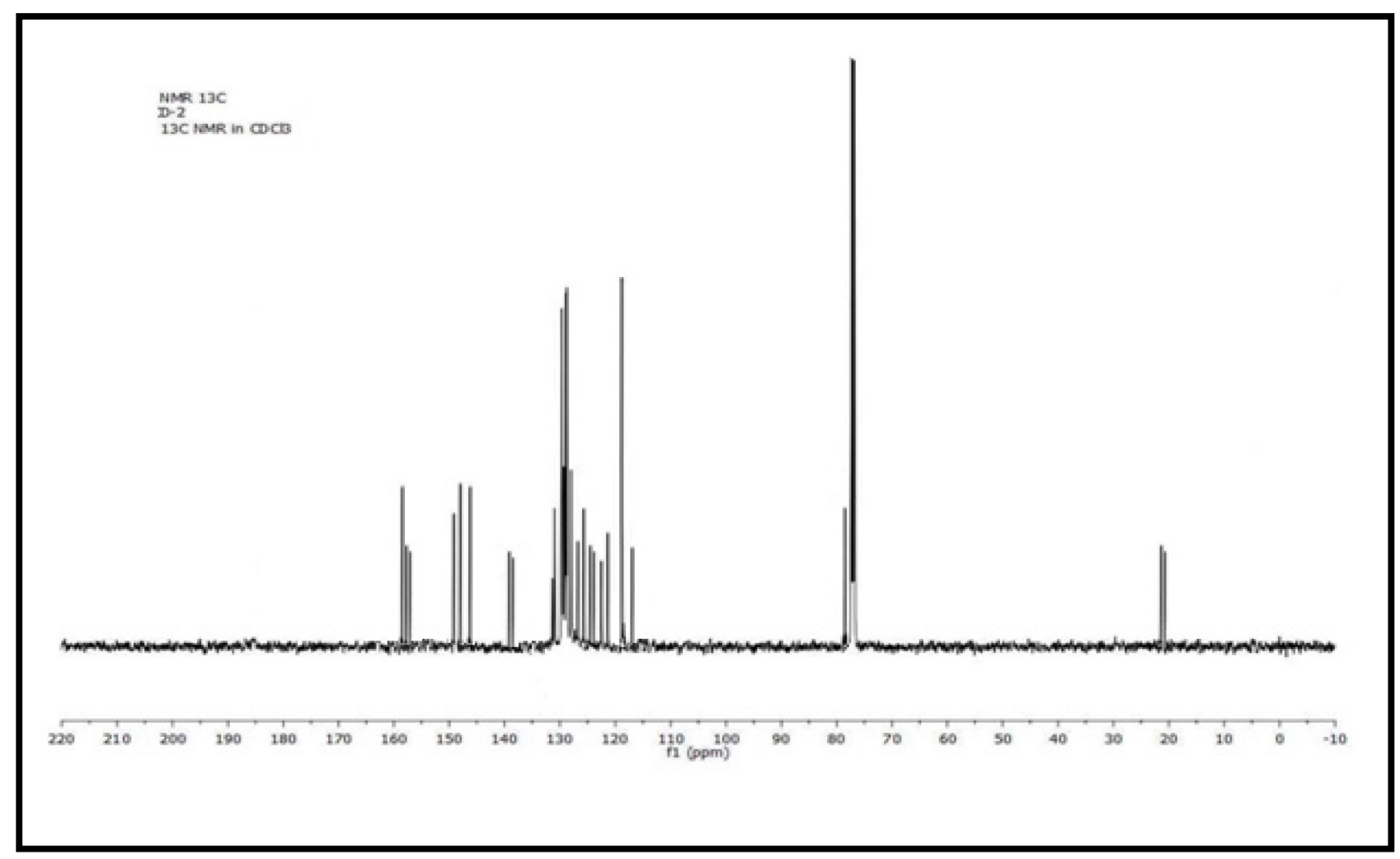

4. ${ }^{13} \mathrm{C}$ NMR of 4-NO ${ }_{2}$ Compound 51

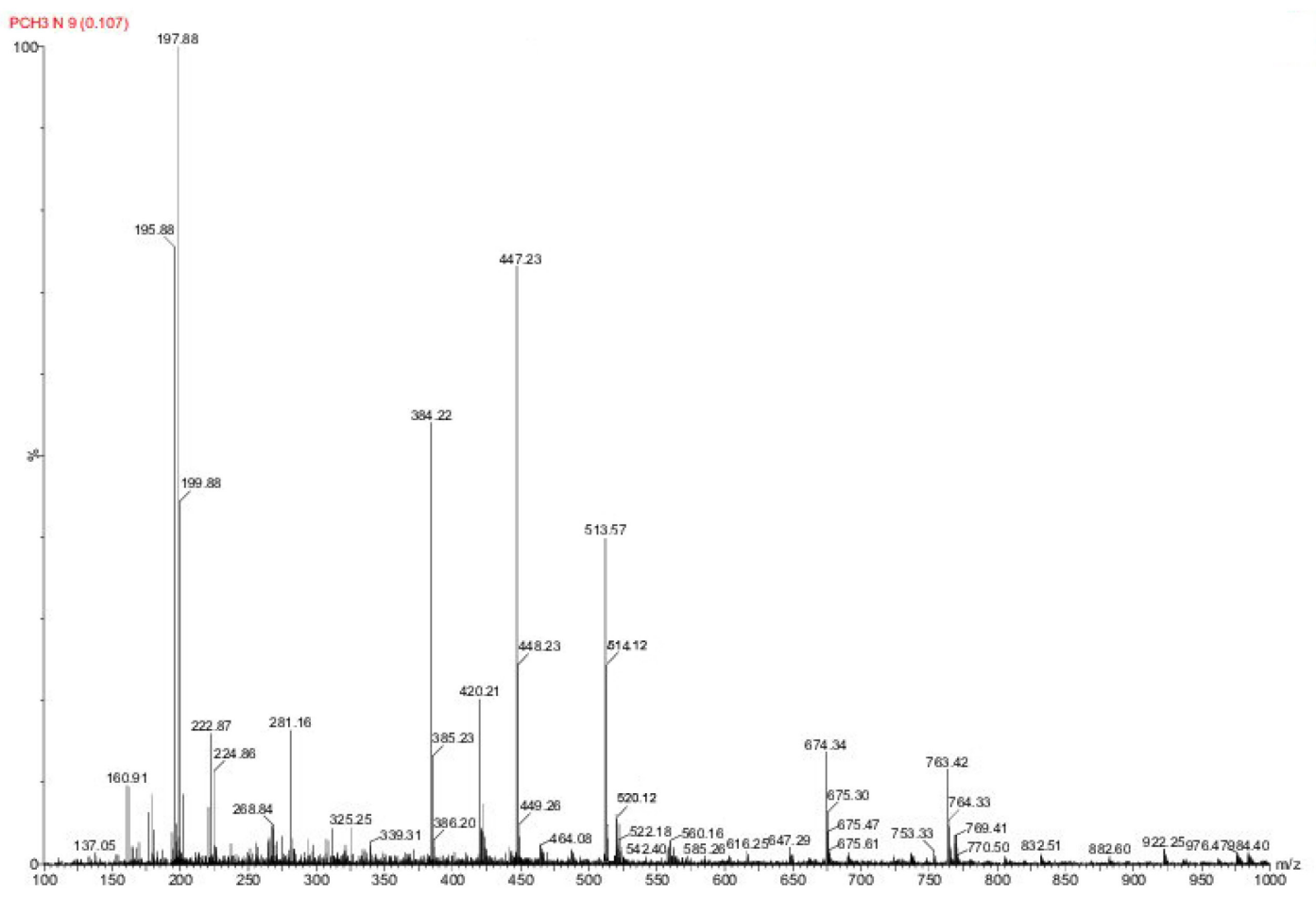

5. Mass of 4-CH $\mathrm{CH}_{3}$ Compound $5 f$ 


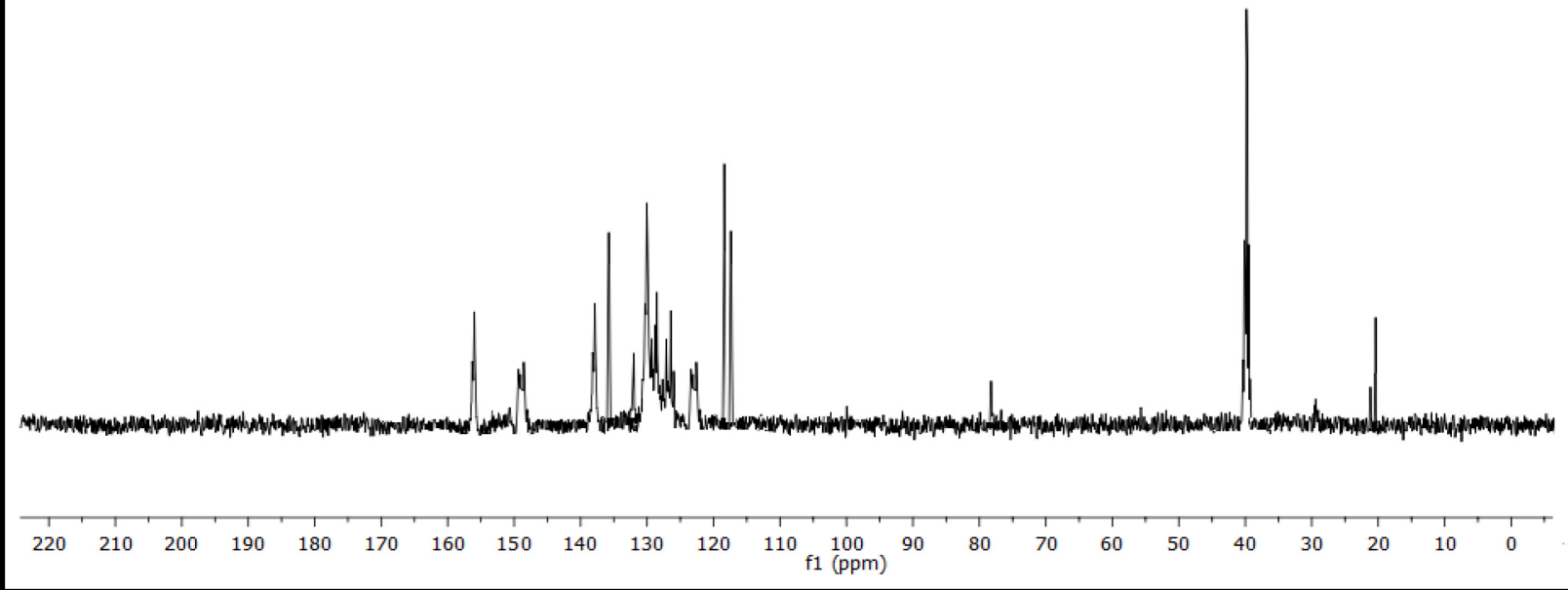

\section{6. ${ }^{13} \mathrm{C}$ NMR of -4- $\mathrm{CH}_{3}$ Compound $5 f$}

\section{References}

[1] P.K. Gupta et al., A metal-free tandem approach to prepare structurally diverse $N$ heterocycles: synthesis of 1,2,4-oxadiazoles and pyrimidinones, New. J. Chem. 38 (2014) 3062-3070.

[2] R. Kharb, R. Kaur, A.K. Sharma, Vistas on antimicrobial potential of novel oxadiazole derivatives in modern medicinal chemistry, European Journal of Biomedical and Pharmaceutical Sciences. 1(2) (2014) 401-420.

[3] S. Distinto et al., Drug design, synthesis, in vitro and in silico evaluation of selective monoaminoxidase B inhibitors based on 3-acetyl-2-dichlorophenyl-5-aryl-2,3-dihydro-1,3,4oxadiazole chemical scaffold, Eur. J. Med. Chem. 108 (2016) 542-552.

[4] R.S. Kumar et al., Anti-inflammatory and antimicrobial activities of novel pyrazole analogues, Saudi. J. Biol. Sci. 23 (2016) 614-620.

[5] D.S. Musmade, S.R. Pattan, S.Y. Manjunath, Oxadiazole a nucleus with versatile biological behaviour, Int. J. Pharm. Chem. 2015. doi:10.7439/ijpc.

[6] D. Allison et al., Synthesis and antimicrobial studies of novel derivatives of 4-(4-formyl-3phenyl-1H-pyrazol-1-yl)benzoic acid as potent anti-Acinetobacter baumannii agents, Bioorg. Med. Chem. Lett. 27 (2017) 387-392.

[7] H. Cheng et al., Synthesis and SAR of heteroaryl-phenyl-substituted pyrazole derivatives as highly selective and potent canine COX-2 inhibitors, Bioorg. Med. Chem. Lett. 16 (2006) 2076-2080. 
[8] M.A.F. Vera-DiVaio et al., Synthesis, antichagasic in vitro evaluation, cytotoxicity assays, molecular modeling, and SAR/QSAR studies of a 2-phenyl-3-(1-phenyl-1H-pyrazol-4-yl)acrylic acid benzylidene-carbohydrazide series, Bioorg. Med. Chem. 17 (2009) 295.

[9] B.P. Chetan, V.V. Mulwar, Synthesis and evaluation of certain pyrazolines and related compounds for their anti tubercular, antibacterial and anti fungal activities, Ind. J. Chem. 44B (2000) 232-237.

[10] R.H. Udupi, A.R. Bhat, K. Krishna, Synthesis and investigation of some new pyrazoline derivatives for their antimicrobial, anti inflammatory and analgesic activities, Ind. J. Heterocycle. Chem. 8 (1998) 143-146.

[11] R.S. Fabiane et al., Hypothermic and antipyretic effects of 3-methyl- and 3-phenyl-5hydroxy-5-trichloromethyl-4,5-dihydro- $1 H$-pyrazole-1-carboxyamides in mice, Eur. J. Pharmacol. 451(2) (2002) 141-147.

[12] K. Ashok, A.S. Sharma, Synthesis of potential quinazolinyl pyrazolines as anticonvulsant agents, Ind. J. Hetero. Chem. 9 (2001) 197.

[13] P. Zoumpoulakis et al., Synthesis of novel sulfonamide-1,2,4-triazoles, 1,3,4-thiadiazoles and 1,3,4-oxadiazoles, as potential antibacterial and antifungal agents. Biological evaluation and conformational analysis studies, Bioorg. Med. Chem. 20 (2012) 1569-1583.

[14] D. Sriram, D. Banerjee, P.J. Yogeeswari, Efavirenz Mannich bases: Synthesis, anti-HIV and antitubercular activities, J. Enzyme. Inhib. Med. Chem. 24(1) (2009) 1-5.

[15] A.S. Aboraia et al., Novel 5-(2-hydroxyphenyl)-3-substituted-2,3-dihydro-1,3,4-oxadiazole-2thione derivatives: promising anticancer agents, Bioorg. Med. Chem. 14 (2006) 1236.

[16] A. Zarghi et al., Synthesis and anticonvulsant activity of new 2-substituted-5-(2benzyloxyphenyl)-1,3,4-oxadiazoles, Bioorg. Med. Chem. Lett. 15 (2005) 1863-1865.

[17] R.A. Rane et al., Synthesis and evaluation of novel 4-nitropyrrole-based 1,3,4-oxadiazole derivatives as antimicrobial and anti-tubercular agents, Eur. J. Med. Chem. 70 (2013) 49-58.

[18] T.M. Kadayat et al., Modified 2,4-diaryl-5H-indeno[1,2-b]pyridines with hydroxyl and chlorine moiety: Synthesis, anticancer activity, and structure-activity relationship study, Bioorg. Chem. 62 (2015) 30-40.

[19] R.M. Mohareb, M.Y. Zaki, N.S. Abbas, Synthesis, anti-inflammatory and anti-ulcer evaluations of thiazole, thiophene, pyridine and pyran derivatives derived from and rostenedione, Steroids. 98 (2015) 80-91.

[20] G. Jose et al., Synthesis, crystal structure, molecular docking and antimicrobial evaluation of new pyrrolo [3,2-c] pyridine derivatives, J. Mol. Struct. 1081 (2015) 85-95.

[21] V.K. Vladimir, G.B. Alicia, Recent developments in the design and synthesis of hybrid molecules base donaminoquinoline ring and their antiplasmodial evaluation, Eur. J. Med. Chem. 44 (2009) 3091-3113.

[22] N.C. Desai et al., Synthesis and antibacterial and cytotoxic activities of new $N-3$ substituted thiazolidine-2,4-dione derivatives bearing the pyrazole moiety, Arch. Pharm. Chem. Life. Sci. 347(7) (2014) 1-10.

[23] N.C. Desai, M.J. Bhatt, Optimized synthesis of novel pyrazole based thiazole derivatives and their antimicrobial evaluation, Int. Lett. Chem. Phys. Astron. 66 (2016) 109-118.

[24] N.C. Desai et al., Synthesis, characterization and antimicrobial screening of hybrid molecules containing benzimidazole-pyrazole and pyridine nucleus, Med. Chem. Res. 21 (2012) 44634472. 
[25] N.C. Desai et al., Dimeric 2-(2-chlorophenyl)-quinazolin-4-ones as potential antimicrobial agents, Med. Chem. Res. 21 (2012) 1127-1135.

[26] N.C. Desai, G.M. Kotadiya, Facile synthesis of pyrazole encompassing pyridyl oxadiazole using conventional and microwave techniques, Curr. Org. Chem. 18 (2014) 2561-2570.

[27] P.C. Hannan, Guidelines and recommendations for antimicrobial minimum inhibitory concentration (MIC) testing against veterinary mycoplasma species, Vet. Res. 31 (2000) 373395.

[28] N.C. Desai et al., Synthesis, antimicrobial and cytotoxic activities of some novel thiazole clubbed 1,3,4-oxadiazoles, Eur. J. Med. Chem. 67 (2013) 54-59.

[29] A. Rattan, Antimicrobials in laboratory medicine, B.I. Churchill Livingstone, New Delhi, 2000, pp. 85-108. 